\title{
Influence of local surface albedo variability and ice crystal shape on passive remote sensing of thin cirrus
}

\author{
C. Fricke ${ }^{1}$, A. Ehrlich ${ }^{1}$, E. Jäkel ${ }^{1}$, B. Bohn ${ }^{2}$, M. Wirth ${ }^{3}$, and M. Wendisch ${ }^{1}$ \\ ${ }^{1}$ Leipzig Institute for Meteorology, University of Leipzig, Leipzig, Germany \\ ${ }^{2}$ Institute for Energy and Climate Research, IEK-8, Forschungszentrum Jülich GmbH, Jülich, Germany \\ ${ }^{3}$ Department of Atmospheric Physics, DLR Oberpfaffenhofen, Germany
}

Correspondence to: C. Fricke (fricke@uni-leipzig.de)

Received: 14 December 2012 - Published in Atmos. Chem. Phys. Discuss.: 11 February 2013

Revised: 13 December 2013 - Accepted: 8 January 2014 - Published: 20 February 2014

\begin{abstract}
Airborne measurements of solar spectral radiance reflected by cirrus are performed with the HALO-Solar Radiation (HALO-SR) instrument onboard the High Altitude and Long Range Research Aircraft (HALO) in November 2010. The data are used to quantify the influence of surface albedo variability on the retrieval of cirrus optical thickness and crystal effective radius. The applied retrieval of cirrus optical properties is based on a standard two-wavelength approach utilizing measured and simulated reflected radiance in the visible and near-infrared spectral region. Frequency distributions of the surface albedos from Moderate resolution Imaging Spectroradiometer (MODIS) satellite observations are used to compile surface-albedo-dependent lookup tables of reflected radiance. For each assumed surface albedo the cirrus optical thickness and effective crystal radius are retrieved as a function of the assumed surface albedo. The results for the cirrus optical thickness are compared to measurements from the High Spectral Resolution Lidar (HSRL). The uncertainty in cirrus optical thickness due to local variability of surface albedo in the specific case study investigated here is below 0.1 and thus less than that caused by the measurement uncertainty of both instruments. It is concluded that for the retrieval of cirrus optical thickness the surface albedo variability is negligible. However, for the retrieval of crystal effective radius, the surface albedo variability is of major importance, introducing uncertainties up to $50 \%$. Furthermore, the influence of the bidirectional reflectance distribution function (BRDF) on the retrieval of crystal effective radius was investigated and quantified with uncertainties below $10 \%$, which ranges below the uncertainty caused by the surface albedo variability. The comparison with the indepen-
\end{abstract}

dent lidar data allowed for investigation of the role of the crystal shape in the retrieval. It is found that if assuming aggregate ice crystals, the HSRL observations fit best with the retrieved optical thickness from HALO-SR.

\section{Introduction}

Passive remote sensing techniques, either satellite-/aircraftbased or from the ground, for retrieving cirrus optical and microphysical parameters are well established (Hong et al., 2007; Eichler et al., 2009; Yang et al., 2005). However, these retrievals are complicated and uncertain, (a) partly due to the complex nonspherical shape of ice crystals and the spatial heterogeneity of cirrus and (b) the impact of surface albedo on the measured signal. The ice crystal shape is an important factor influencing the radiative properties of cirrus (Eichler et al., 2009; Wendisch et al., 2005) because the single-scattering properties of ice crystals differ from those of spheres. As shown in Key et al. (2002), ice crystal shape has an influence on the retrieved cirrus optical properties, with maximum shape-induced differences of up to $60 \%$ for $\tau$ and about $20 \%$ for $r_{\text {eff }}$ demonstrating the high sensitivity of the retrieval to the choice of the assumed ice particle model (Zhang et al., 2009). Furthermore, for optically thin cirrus it is hard to distinguish between the solar radiation scattered by the cirrus and that originating from the atmosphere, aerosol particles and Earth's surface. Compared to the radiation reflected by thin cirrus, the portion reflected by the surface and atmosphere may dominate the signal measured by the satellite (Gao et al., 1998). Optically thin cirrus is widespread 

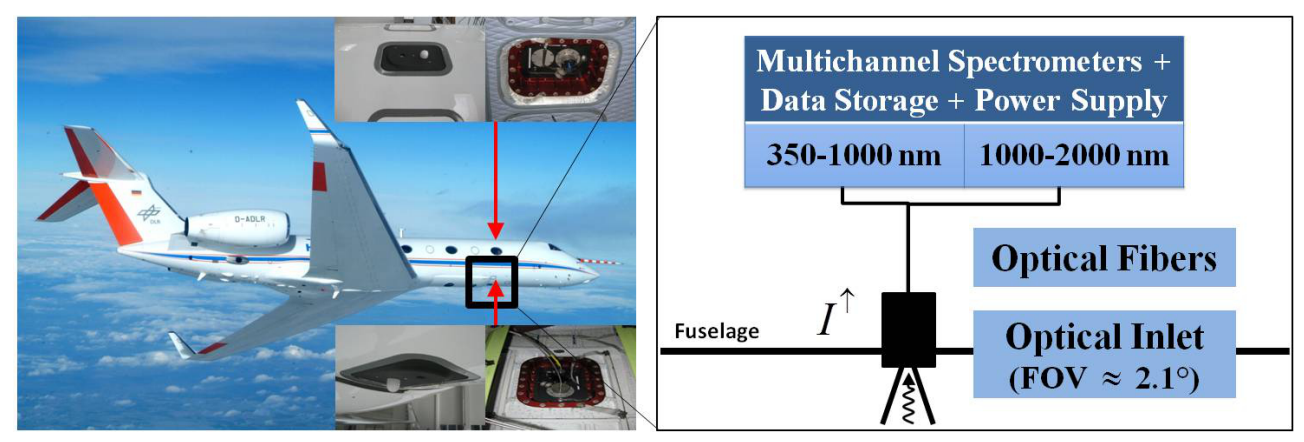

Fig. 1. Illustration of the HALO-SR measurement setup on HALO consisting of an optical inlet $\left(\mathrm{FOV} \approx 2.1^{\circ}\right)$, spectrometers and a bifurcated optical fiber that transmits the signal from the optical inlet to two Zeiss multispectral spectrometers monitoring from 350 to $2000 \mathrm{~nm}$.

over the globe, as shown by Sassen et al. (2008), and may remain undetected over highly reflecting ground (Gao et al., 1998). Even for optically thick cirrus, the uncertainties in retrieving cloud optical properties may increase for heterogeneous surfaces as most models assume a homogeneous surface albedo. To increase the accuracy of the retrieval results for optical thin cirrus and to avoid producing large errors in the retrieved parameters, an accurate estimation of the surface albedo has to be used in the retrieval algorithm, especially over land surfaces (Rolland et al., 2000). However, surface albedo data are not always available, and heterogeneous surfaces can make correct allocation of surface albedo impossible.

To quantify the influence of surface albedo and crystal shape we have performed airborne measurements of solar radiation in the solar spectral range $(400-2000 \mathrm{~nm})$ reflected from cirrus. The spectral radiances were measured by HALO-SR (HALO-Solar Radiation) onboard the new German research aircraft HALO (High Altitude and Long Range Research Aircraft) during the Techno Mission in October and November 2010. The spectroradiometer system HALOSR was installed on HALO to measure upward spectral radiances in combination with an instrument for measurements of the spectral actinic flux density. In this work, the collected spectral radiance data are used in combination with one-dimensional (1-D) radiative transfer calculations to retrieve cirrus properties (effective radius and optical thickness) as well as information on the ice crystal shape and spatial distribution of cirrus. Based on $1 \mathrm{~km}$ resolution surface albedo maps derived from MODIS (Moderate Resolution Imaging Spectroradiometer), the retrieval is performed assuming the surface albedo in four different approaches to quantify the effect of surface albedo local variability on the retrieved cirrus properties. To investigate the influence of the ice crystal shape, the retrieval is performed assuming different ice crystal parametrizations. The retrieved cirrus properties are compared with measurements of cirrus optical thickness from the high-spectral-resolution lidar (HSRL) WALES (Water vApour Lidar Experiment in Space) simultaneously operated on HALO (Esselborn et al., 2008; Wirth et al.,
2009; Piironen and Eloranta, 1994). While HALO-SR performs passive measurements in the solar spectral range, the HSRL is an active instrument providing vertically resolved and surface-albedo-independent data that can be analyzed without radiative transfer simulations. This allows deriving cirrus optical thicknesses not affected by the surface albedo. In contrast, HALO-SR, like most passive satellite-based sensors, measures vertically unresolved and relies on accurate assumptions of crystal shape and surface albedo in the radiative transfer.

\section{Measurements and methods}

\subsection{Airborne remote sensing of cirrus using HALO-SR and HSRL}

Based in Oberpfaffenhofen, Germany, HALO performed six flights during the Techno Mission within German airspace between 27 October and 5 November 2010. Reflected solar spectral radiance above cirrus was measured aboard HALO using the spectroradiometer system HALO-SR consisting of one downward-looking optical inlet (field of view, $\mathrm{FOV} \approx 2.1^{\circ}$ ), one bifurcated optical fiber and two spectrometers (fixed gratings, photodiode array detectors) (see Fig. 1). The system is similar to the Spectral Modular RAdiation measurement sysTem (SMART)-Albedometer introduced by Wendisch et al. (2001) and further developed by Bierwirth et al. (2010). It monitors a spectral range from 350 to $2000 \mathrm{~nm}$ with a spectral resolution of 2 to $3 \mathrm{~nm}$ (full width at half maximum, FWHM) for the wavelength range between 350 and $1000 \mathrm{~nm}$ and FWHM of 9 to $16 \mathrm{~nm}$ for the near-infrared (NIR) wavelength range 1000 to $2000 \mathrm{~nm}$. A measurement uncertainty of $5 \%$ has to be considered, which is mostly caused by the calibration procedure using a certified diffuse radiation source traceable to NIST (National Institute of Standards and Technology). An additional $3 \%$ is caused by a transfer-calibration procedure. The nadir-viewing optical inlet receives the radiances reflected by clouds, atmosphere and surface within its FOV, which 


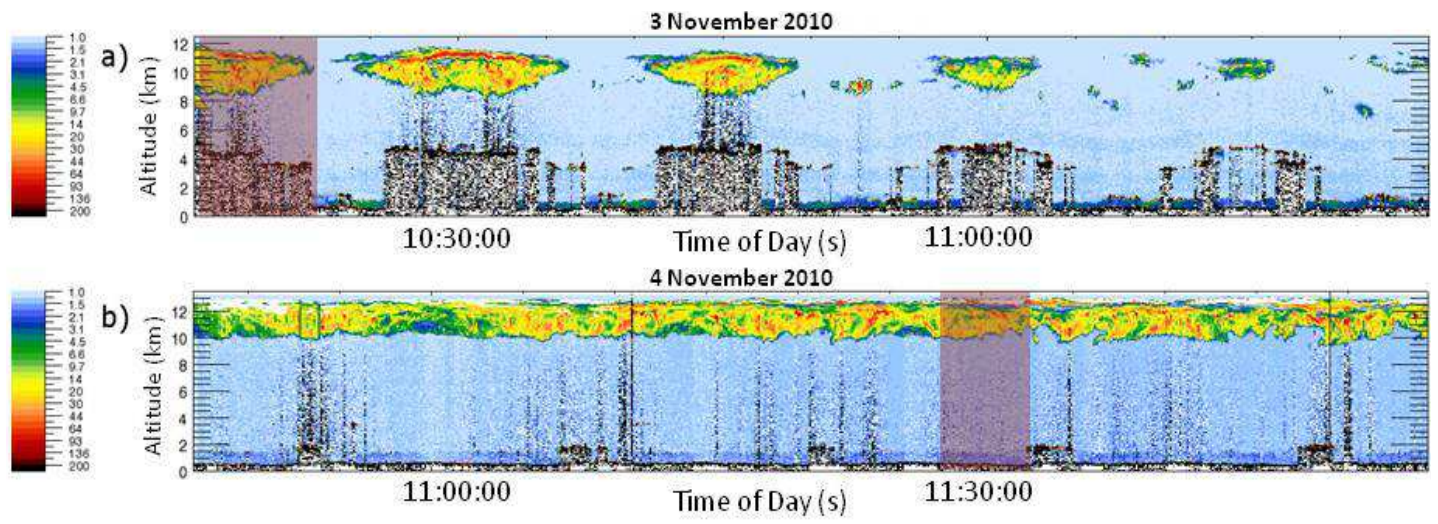

Fig. 2. (a) Backscatter ratio from HSRL for a $75 \mathrm{~min}$ time frame of the flight of 3 November 2010 showing two descending cloud layers alternating with cloud-free areas. One cloud layer is situated between 9 and $13 \mathrm{~km}$ altitude, and a second one below $5 \mathrm{~km}$ altitude. The investigated time series is highlighted in red. (b) Backscatter ratio from HSRL for a 75 min time frame of the flight of 4 November 2010 showing cirrus layer between 9 and $13 \mathrm{~km}$ altitude. The investigated time series is highlighted in red. Time is given in UTC.

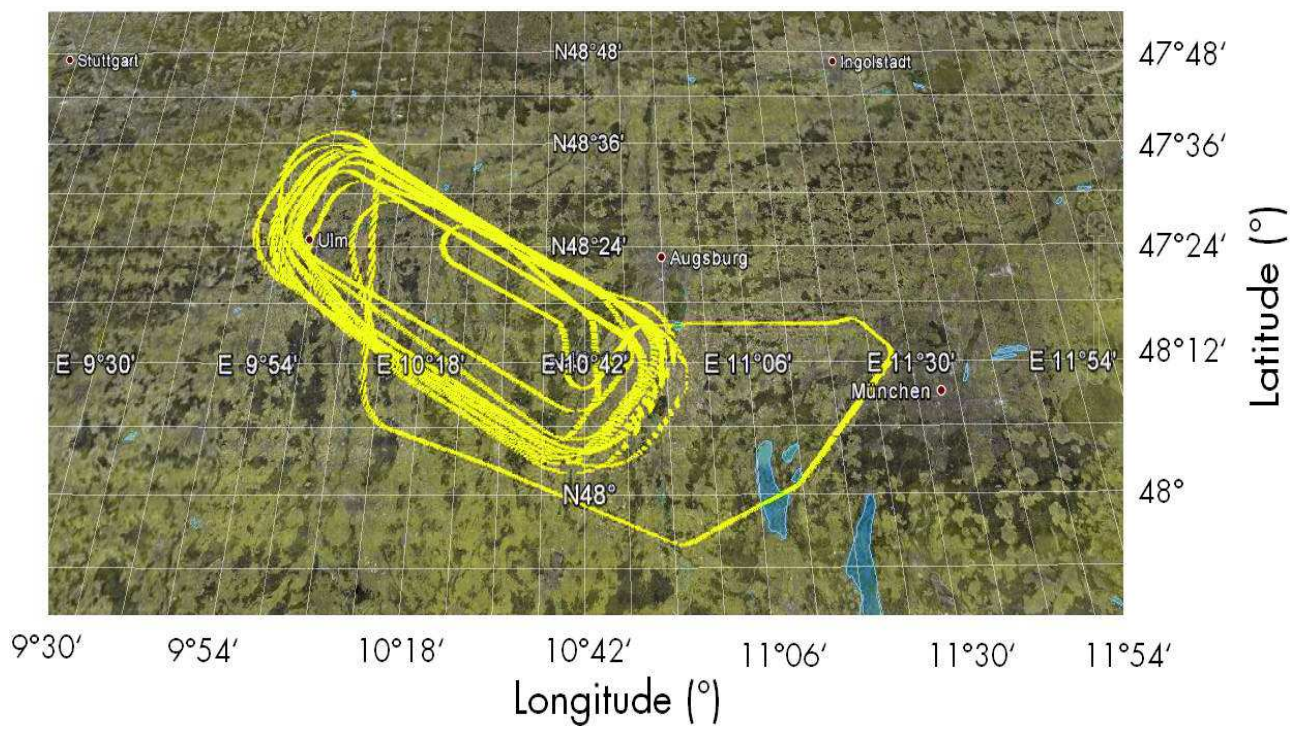

Fig. 3. Flight track of 3 November 2010 over the measurement area in southern Germany (illustrated using Google Earth).

corresponds to a footprint at the surface of $1 \mathrm{~km}^{2}$ including the flight movement for $14 \mathrm{~km}$ flight altitude.

In particular the measurements on 3 and 4 November 2010 are analyzed. The time series of HSRL profiles of backscatter ratio are presented in Fig. 2 for these two days, with investigated time frames highlighted in red. HSRL backscatter ratio is given at $532 \mathrm{~nm}$ (Piironen and Eloranta, 1994). The data of 4 November 2010 recorded at an altitude of about $14 \mathrm{~km}$ reveal a homogeneous cirrus between 9 and $13 \mathrm{~km}$ between 11:00 and 11:30 UTC (Fig. 2b). The possibility of water and/or mixed-phase clouds is excluded because cirrus is present above $8 \mathrm{~km}$ altitude only where the measured temperatures are well below $235 \mathrm{~K}$. The flight pattern and flight altitude (about $14 \mathrm{~km}$ ) of the second flight were almost identical to the flight of 4 November (Fig. 2a). How- ever, on 3 November 2010 the cirrus field was less homogeneous than on 4 November 2010. Two separate cloud layers below the plane were partly present on November 3 . In areas with cirrus a second cloud layer was situated at about $5 \mathrm{~km}$ altitude below the cirrus between 8.5 and $13 \mathrm{~km}$. The flight pattern (Fig. 3) covers an area of approximately $3600 \mathrm{~km}^{2}$. Eight consecutive rectangular patterns with leg lengths of approximately 20 and $50 \mathrm{~km}$ were performed. Time series of radiance measurements at two wavelengths $(650 \mathrm{~nm}$ and $1646 \mathrm{~nm})$ are shown in Fig. 4 for 3 November 2010 between 11:05 and 11:15 UTC. They demonstrate fluctuations in the measured radiance that results either from the heterogeneity of the surface albedo or from the variation of cirrus properties (including the second cloud layer below). The $650 \mathrm{~nm}$ wavelength radiance ranges between 


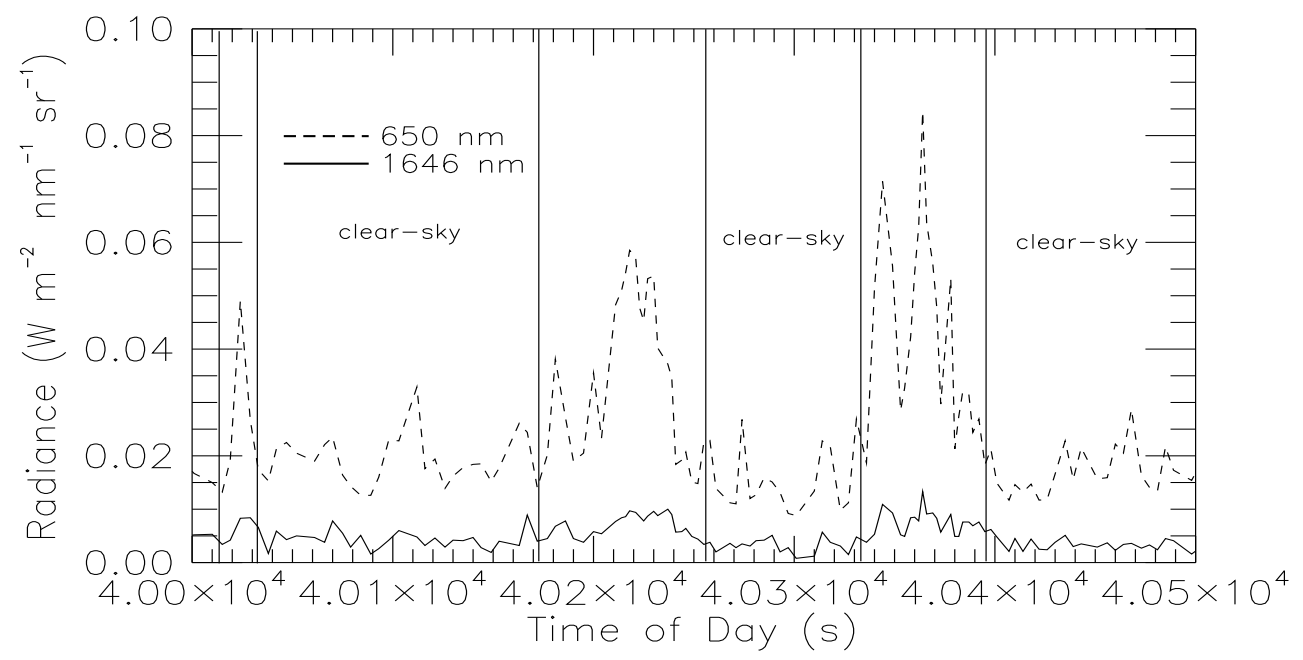

Fig. 4. Example time series (UTC) of HALO-SR radiance measurement reflected by the cirrus on 3 November 2010 for two wavelengths.

0.02 and $0.08 \mathrm{~W} \mathrm{~m}^{-2} \mathrm{~nm}^{-1} \mathrm{sr}^{-1}$ for the cloudy regions and 0.02 and $0.04 \mathrm{~W} \mathrm{~m}^{-2} \mathrm{~nm}^{-1} \mathrm{sr}^{-1}$ during clear-sky conditions. For $1646 \mathrm{~nm}$ the values are generally lower varying between 0.002 and $0.008 \mathrm{~W} \mathrm{~m}^{-2} \mathrm{~nm}^{-1} \mathrm{sr}^{-1}$ with slightly higher values in the cloudy cases. As the optical inlet is fixed to the aircraft fuselage, aircraft roll and pitch movements cause a deviation of the viewing direction from exactly nadir. To minimize errors due to nadir misalignment, data with roll and/or pitch angles of the airplane larger than $3^{\circ}$ were excluded from the data analysis.

\subsection{Surface albedo distributions from MODIS}

MODIS satellite data of surface albedo from 29 October 2010 are used to assemble frequency distributions of surface albedo representative for the area covered during the measurements, as it is the most contemporary data available. The surface albedo is determined by an interpolation between the black-sky (direct beam) albedo and white-sky (completely diffuse) albedo as a function of the solar zenith angle and the fraction of diffuse skylight, which in turn is a function of optical depth based on the formula given at Schaaf et al. (2002). The MODIS surface albedo (Schaaf et al., 2002) is extracted for different areas representing local surface albedo variability, allowing for quantifying the influence of the surface albedo variability. A first approach includes the surface albedo variability covered by a rectangular area of approximately $3600 \mathrm{~km}^{2}$ containing the whole flight track (Fig. 3). In a second approach, the surface albedo is extracted along the flight track within a $100 \mathrm{~km}^{2}$ footprint area below the plane. In a third approach, the mean surface albedo within a $10 \mathrm{~km}^{2}$ footprint area below the plane is allocated for each measurement. The mean surface albedo value of the entire $3600 \mathrm{~km}^{2}$ area is used as further reference in a fourth approach. Corresponding to the two retrieval wavelengths, surface albedo data of MODIS band B1 (620 to $670 \mathrm{~nm})$ and B6 (1628 to
$1652 \mathrm{~nm}$ ) were used. In this regard, the investigated measurement data are spectrally convolved with the spectral response function of the MODIS bands. The footprint of HALO-SR results from combining the FOV of the optical inlet and the flight distance covered during the sampling time (1 to $3 \mathrm{~s}$ ). This results in an average footprint size of about $1 \mathrm{~km}^{2}$. Figure $5 \mathrm{a}$ and $\mathrm{b}$ display the albedo frequency distribution of both bands B1 and B6 for the first approach. Figure 6a and b display the same for the second approach. The two-dimensional (2-D) frequency distribution of the second approach shows possible pairs of surface albedo for the B1 band between 0.025 and 0.09 and for the B6 band between 0.08 and 0.23 , with a maximum probability of about 0.07 and 0.2 and standard deviations of 0.018 and 0.04 , respectively. As expected, the 2-D distribution of the rectangle-shaped area of approach 1 reveals a larger variation of the $\mathrm{B} 1$ and $\mathrm{B} 6$ surface albedo; however the albedo with maximum probability of 0.08 (B1) and 0.17 (B6) and standard deviations of 0.04 (B1) and 0.06 (B6) show similar values despite the larger variability of surface albedo, which may have been caused by an increasing number of different surface types sampled during the flight.

\section{Retrieval of cirrus properties}

\subsection{Standard retrieval}

Cirrus optical properties were retrieved using the standard method by Nakajima and King (1990) utilizing the four surface albedo approaches described in Sect. 2.2. This standard retrieval was initially applied to warm liquid water clouds consisting of spherical droplets, but can be adapted to airborne cirrus measurements (Eichler et al., 2009) if appropriate shape information is included. The standard retrieval method utilizes the reflected radiance at a nonabsorbing wavelength $(650 \mathrm{~nm})$ and at an absorbing wavelength 

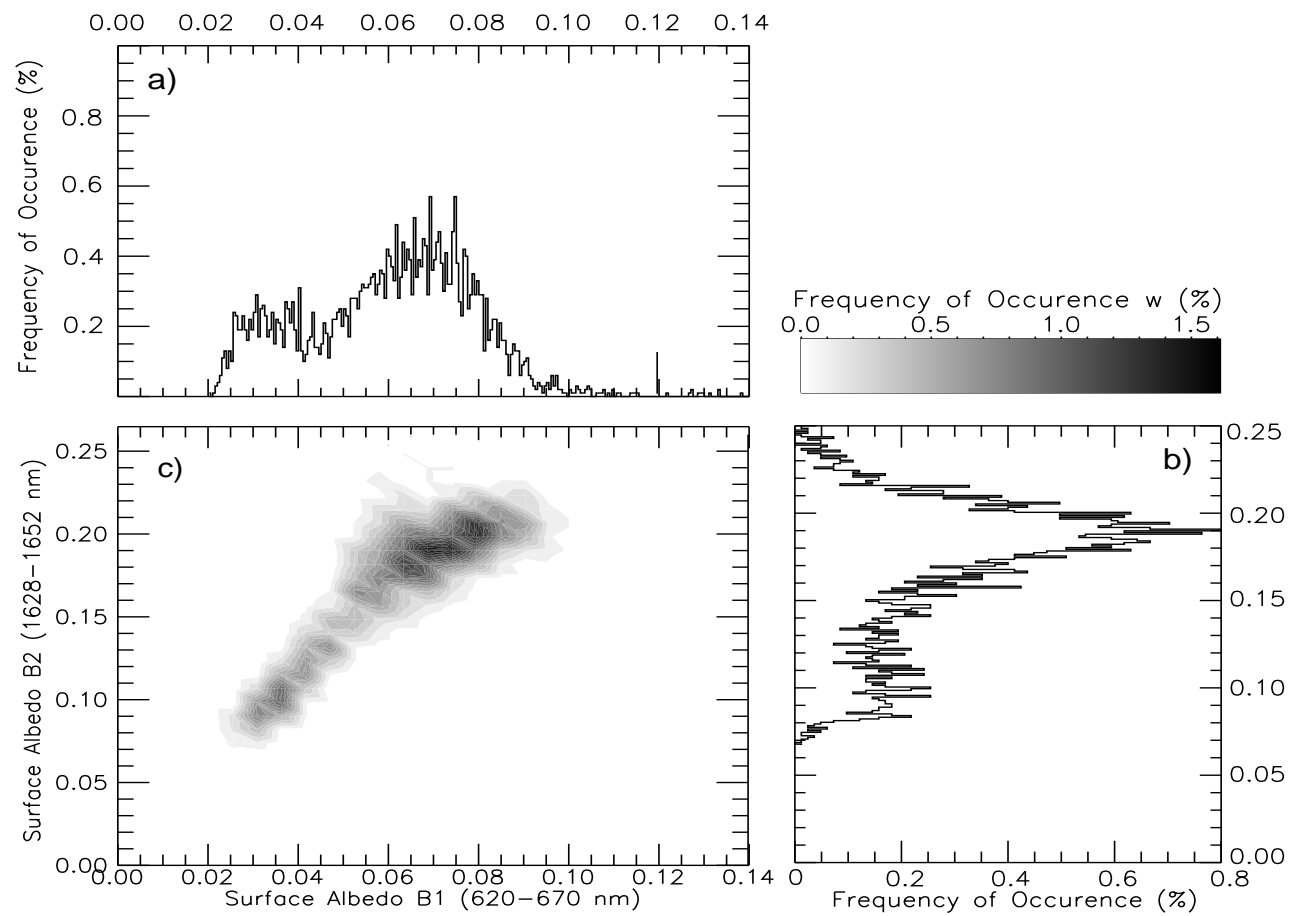

Fig. 5. Two-dimensional (2-D) frequency distribution of the combined B1 and B6 surface albedo from MODIS for the rectangle-shaped area including the flight track covering an area of approximately $3600 \mathrm{~km}^{2}$ (approach 1). Each value stands for a surface albedo pair with a bin size of 0.001. In panels (a) and (b) the separate B1 and B6 distributions are displayed.
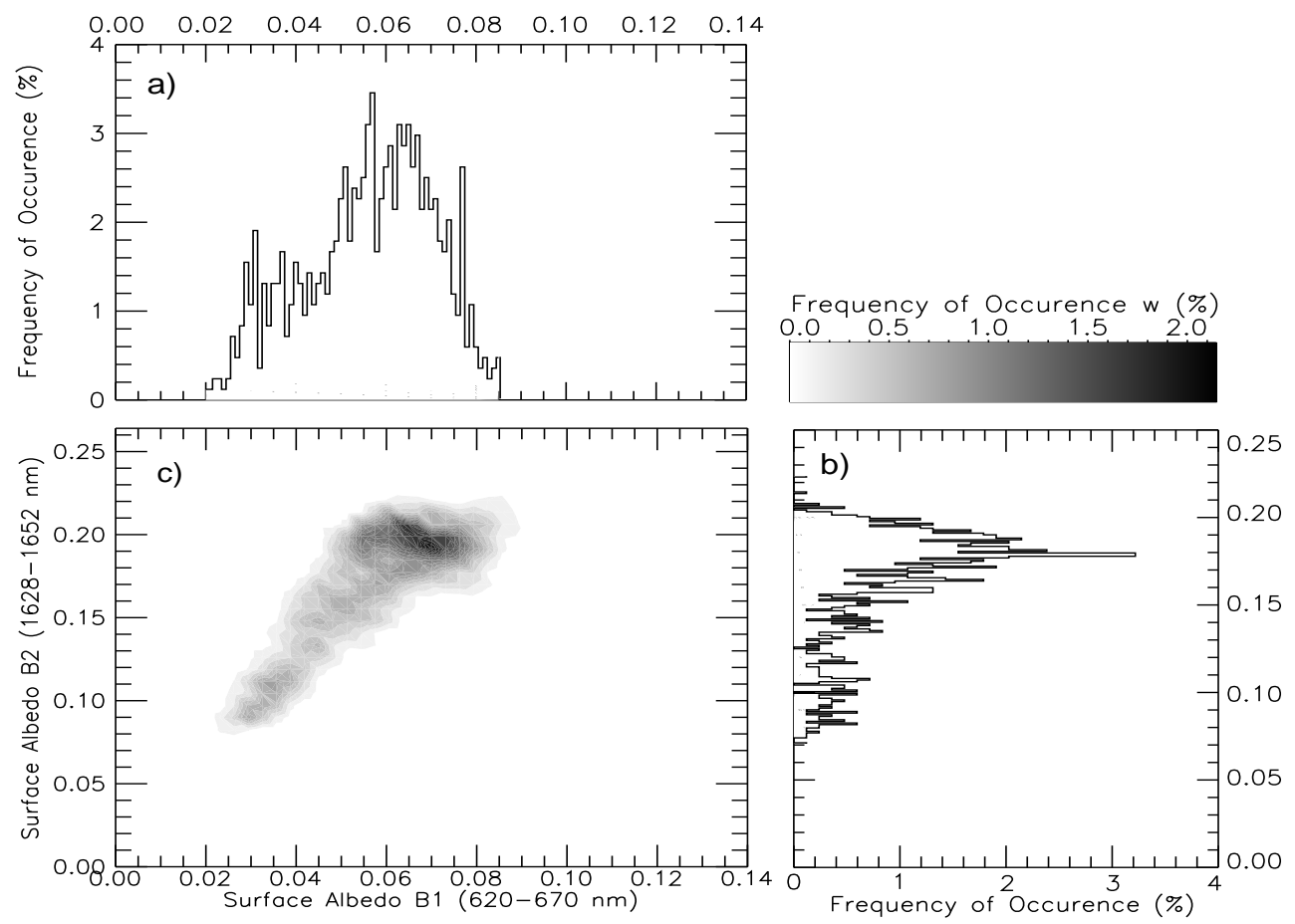

Fig. 6. The same as Fig. 5 but covering a footprint of $100 \mathrm{~km}^{2}$ (approach 2). Each value stands for a surface albedo pair with a bin size of 0.001. In panels (a) and (b) the separate B1 and B6 distributions are displayed. 

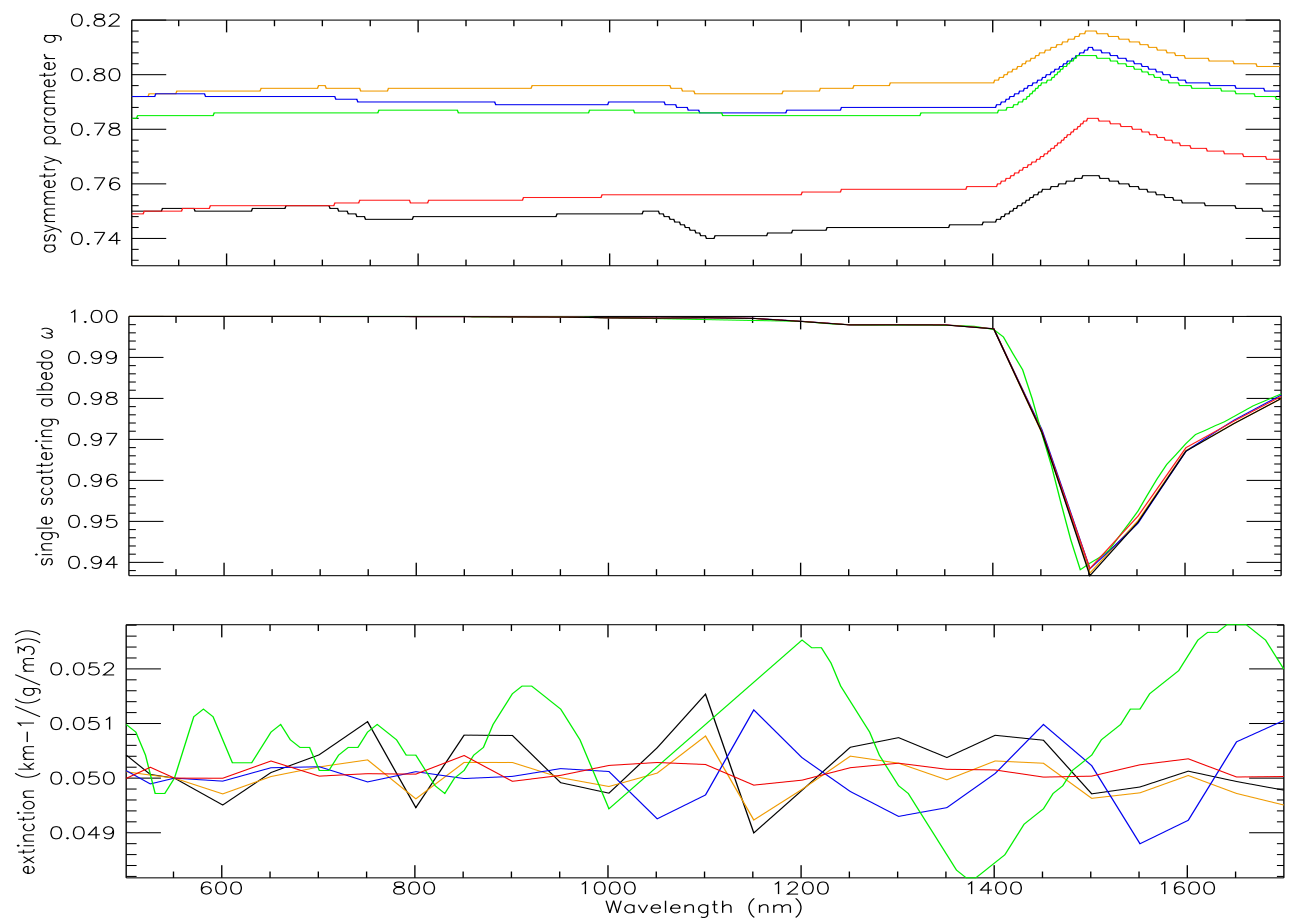

Fig. 7. Parametrized spectral optical properties for a mixture of ice crystals (green), droxtals (blue), aggregates (red), solid columns (black) and hollow columns (yellow).

$(1646 \mathrm{~nm})$. Using radiative transfer calculations, lookup tables of reflected radiances at these two wavelengths are created for different combinations of optical and microphysical properties. In this way the cirrus optical thickness $(\tau)$ and the effective radius $\left(r_{\text {eff }}\right)$ are prescribed in the calculations. The corresponding simulated radiance pairs are matched with the measured radiances. The optical thickness is defined as

$\tau=\int_{z_{1}}^{z_{2}} b_{\text {ext }}(z) \mathrm{d} z ;$

$b_{\text {ext }}$ describes the volumetric extinction coefficient in units of $\mathrm{m}^{-1}$ integrated over the cirrus vertical extend $\left(z_{1}\right.$ representing cloud base and $z_{2}$ cloud top). It determines the fraction of radiation that is attenuated (scattered, absorbed) by the cirrus. The second retrieved parameter is the effective radius of cirrus $r_{\text {eff }}$, defined by

$r_{\text {eff }}=\frac{3}{4} \frac{\mathrm{V}}{\mathrm{A}}$,

with $\mathrm{V}$ as the total volume and $\mathrm{A}$ as the total area cross section of the crystal number size distribution. Ice crystals exhibit different shapes and each shape gives rise to difficulties in measuring the particle size distribution (PSD). As the volume and cross section are integrated over the particle size distribution, several mathematical expressions are suggested based on observations. For the 1-D radiative transfer simulations the ice crystal shape is considered by parametrizations, including complete phase functions simulating a mixture of different ice crystal habits for measurements of ice clouds in midlatitudes as well as in the tropics and subtropics (Baum et al., 2007). Baum et al. (2005) discuss details on the measurements from which the data are derived. Here each PSD is parametrized in the form of a gamma distribution defined by

$n\left(D_{\max }\right)=N_{0} \cdot D_{\max }^{\mu} \cdot \mathrm{e}^{-\lambda D_{\max }}$,

where $D_{\max }$ is the particle maximum dimension, $\mathrm{n}(\mathrm{D})$ is the particle concentration per unit volume. $\mathrm{N}_{0}$ is derived from the intercept, $\lambda$ is derived from the slope and $\mu$ is derived from the dispersion on a log-log plot of this equation (Heymsfield et al. 2002).

Figure 7 displays the parametrized spectral optical properties for the mixture as well as for single crystal habits in the spectral range from 500 to $1700 \mathrm{~nm}$, showing basic differences in the asymmetry parameter and extinction coefficient between the single crystal habits as well as in simulation of a mixture.

For the 1-D simulations the radiative transfer calculation package libRadtran (Mayer and Kylling, 2005) was used. The calculations are based on the DISORT II solver using 16 terms in the scattering phase function; additionally, a midlatitude winter atmosphere was used, and the parametrization for molecular absorption is treated with LOWTRAN (Ricchiazzi et al., 1998). The atmospheric aerosol is derived from HSRL measurements of the extinction coefficients during clear-sky 

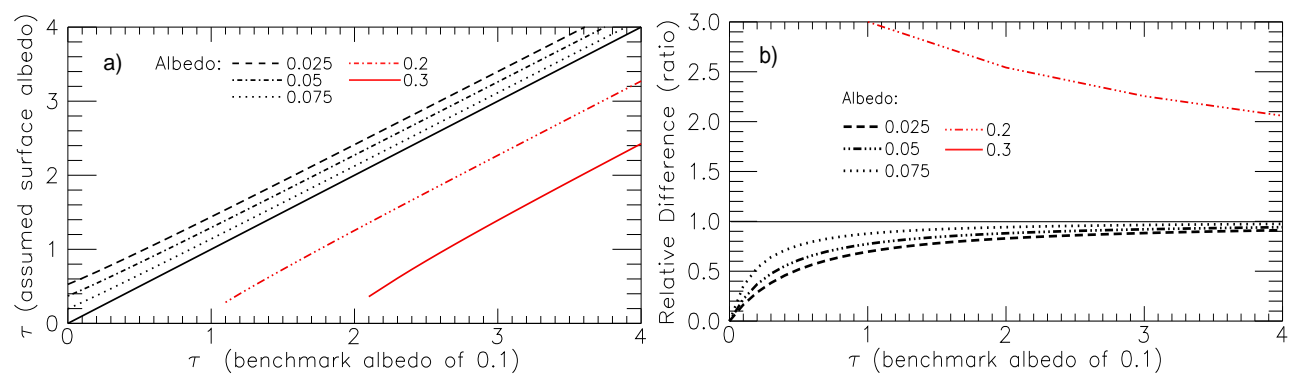

Fig. 8. (a) Absolute and (b) relative difference (ratio) between the result of a retrieval of $\tau$ and the given values derived by creating a cirrus with optical thicknesses between 0 and 4 , a fixed surface albedo of 0.1 and the same retrieval assuming a surface albedo of $0.025,0.05,0.075$, 0.2 and 0.3 .
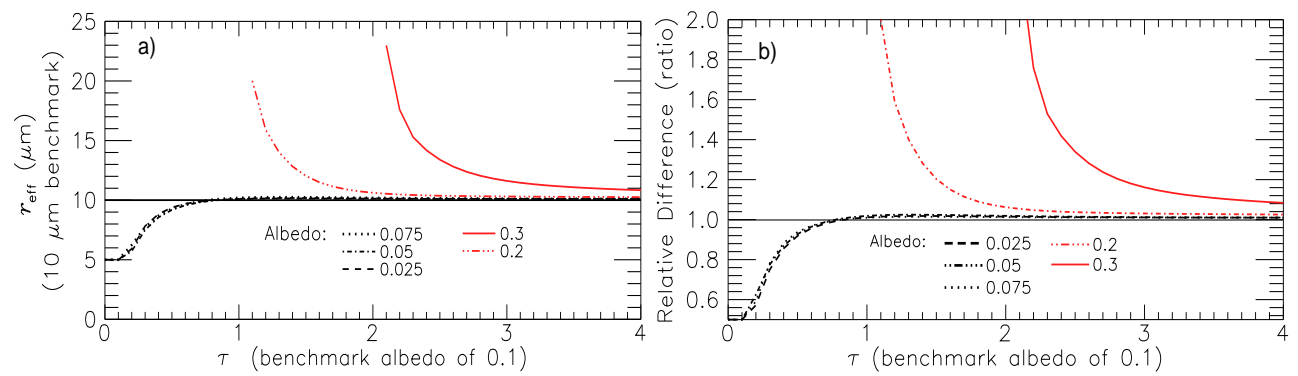

Fig. 9. (a) Absolute and (b) relative difference (ratio) between the result of a retrieval of $r_{\text {eff }}$ and the given values and a benchmark crystal size of $10 \mu \mathrm{m}$ derived by creating a cirrus with optical thicknesses between 0 and 4 , a fixed surface albedo of 0.1 and the same retrieval assuming a surface albedo of $0.025,0.05,0.075,0.2$ and 0.3 .

conditions. Wirth et al. (2009) discuss the retrieval method in detail.

\subsection{Sensitivity of retrieval of cirrus properties over a homogeneous surface}

For a plane-parallel geometry, the influence of a homogeneous surface albedo on the reflected radiance above the cirrus and the corresponding retrieval results is quantified using radiative transfer simulations. Optically thick cirrus causes high extinction. Thus, the reflected radiance mainly results from scattering near the cloud top. In this case the surface albedo is expected to have a minor impact on both the reflected radiance above cirrus and the retrieved cirrus properties. The situation for optically thin cirrus is more complex. The reduced extinction of optically thin cirrus leads to an increased portion of transmitted downward radiation passing the cloud and being reflected back upward by the surface. The received upward-directed radiance above the cirrus consists of contributions reflected by the cirrus and the surface. Atmospheric scattering adds to the upward radiance as well, but can be neglected compared to the cirrus and surface contributions, especially for the wavelength $(650 \mathrm{~nm})$ considered here.

The influence of a homogeneous surface on the retrieval was studied by varying the values of surface albedo spectrally homogeneous in the radiative transfer simulations to
$0.025,0.05,0.075,0.1,0.2$ and 0.3 , covering most of the surface types except ice-based types and disregarding the spectral characteristics. For each surface albedo, simulations of reflected radiance are performed and used to create lookup tables for the retrieval. The values of simulations using the albedo of 0.1 were used as benchmark representing hypothetical radiance measurements. These data were used as retrieval input and applied to all calculated lookup tables. Figure 8 illustrates the retrieved values optical thickness for different values of assumed surface albedo. Here, a constant value of $10 \mu \mathrm{m}$ was used for $r_{\text {eff }}$.

The cirrus optical thickness was varied $(0 \leq \tau \leq 4)$. If surface albedo values of $0.075,0.050$ or 0.025 are assumed instead of 0.1 (benchmark), the retrieved $\tau$ show a significant overestimation, as seen in Fig. 8a. The ratio between true and retrieved optical thickness converges to 1 with increasing optical thickness and reaches a value of 0.8 at $\tau=0.6$ for an albedo deviation of $0.025, \tau=1.4$ for an albedo deviation of 0.05 , and $\tau=2$ for an albedo deviation of 0.075 (see Fig. 8b). Assumptions of surface albedo values larger than the benchmark value ( 0.2 and 0.3 ) lead to underestimations of the cirrus optical thickness. As a consequence, no cirrus can be retrieved until $\tau=1.1$ for the surface albedo assumption of 0.2 and $\tau=2.1$ for the assumption of the surface albedo of 0.3 . For optically thicker clouds, the relative differences reduce independently of the surface albedo 


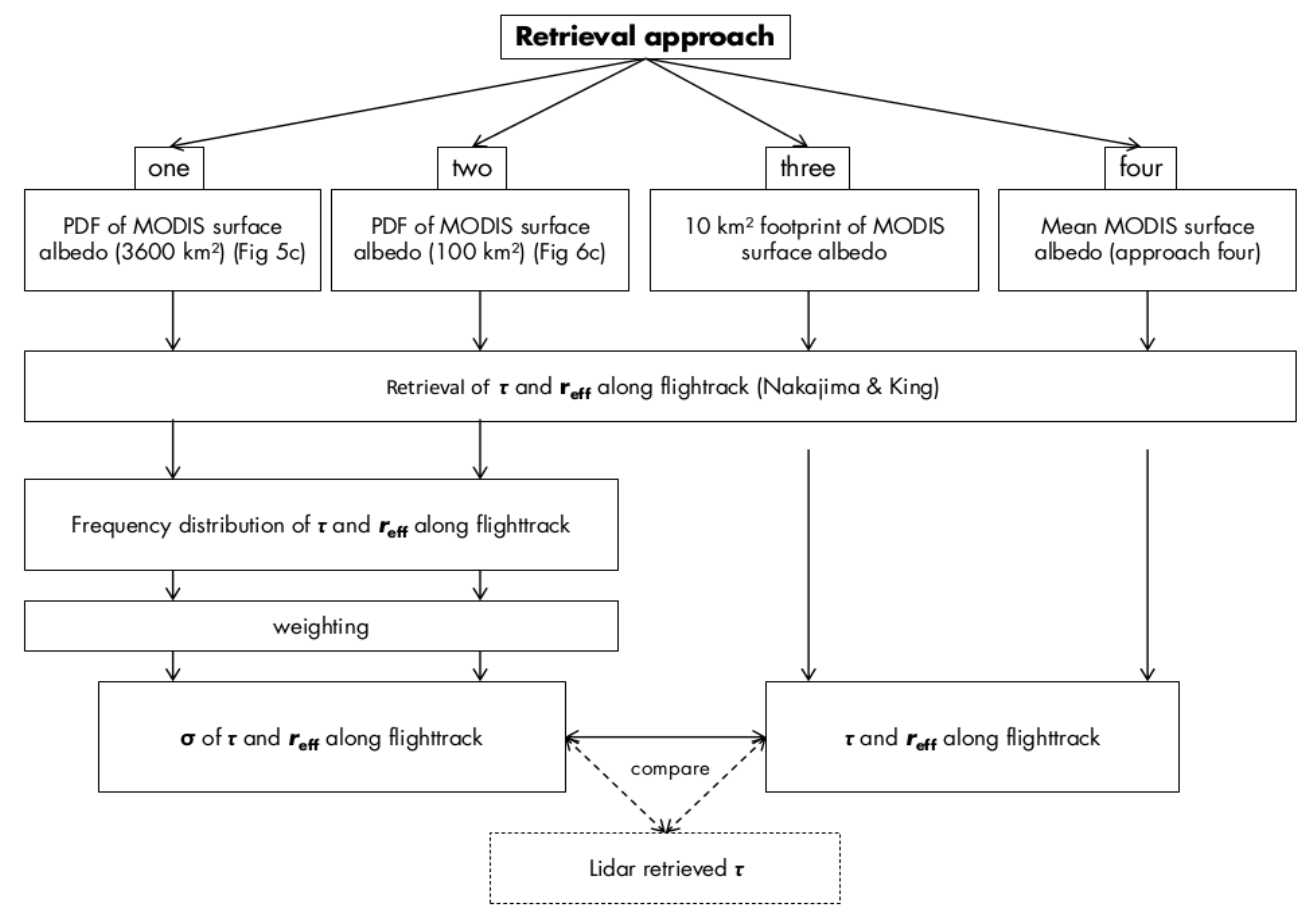

Fig. 10. Schematic sketch illustrating the different retrieval approaches quantifying the influence of surface albedo variabilities.

assumptions, leading to a negligible influence of the surface albedo for optically thick clouds. Contrary to this, the difference of the retrieved $\tau$ offers an exponential increase for optically thin cirrus $(\tau \leq 1)$ in this example. In addition to that, an increasing deviation of retrieved cirrus optical thickness with increasing deviation of surface albedo is obvious in both Fig. 8a and b. For the retrieval of $r_{\text {eff }}$ the same assumptions, except for an additional crystal size benchmark of $10 \mu \mathrm{m}$, are used (Fig. 9). The surface albedo influence also influences the $r_{\text {eff }}$ retrieval, although it converges more strongly to the benchmark value with increasing cirrus optical thickness. Particularly the differences between the surface albedo assumptions that are smaller than the benchmark value of 0.1 show only slight variations among each other. However, for thin optical thicknesses, an exponential increase in deviation is obvious as well.

\subsection{Four approaches of cirrus retrieval}

The cirrus retrieval approaches 1 and 2 (Section 2.2.) are based on using the 2-D surface albedo frequency distribution (Figs. 5c and 6c), where each dimension represents one wavelength, instead of using fixed surface albedo values in the retrieval of $\tau$ and $r_{\text {eff. }}$ As a result 2-D frequency distributions of $\tau$ and $r_{\text {eff }}$ are obtained, as well as respective mean values $\bar{\tau}, \bar{r}_{\text {eff }}$ and standard deviations $\sigma_{\tau}, \sigma_{r_{\text {eff }}}$ for each measurement point along the flight track. Using the surface albedo frequency distributions presented in Fig. 5c (approach 1) and 6c (approach 2), the retrieval of $\tau$ and $r_{\text {eff }}$ was per- formed for each albedo combination and measurement point. In a first step, 1-D radiative transfer calculations are carried out for each possible B1 and B6 surface albedo combination of the surface albedo frequency distribution (Fig. 6a and 6b) and respective grids of combined $\tau$ and $r_{\text {eff }}$ corresponding to the retrieval of Nakajima and King (1990). In a second step, for each radiance measurement along the flight track, cirrus parameters are retrieved using all available lookup tables. All different results (different lookup tables) were weighted with the normalized frequency distribution of the corresponding albedo (Fig. 5c, 6c) for the approaches 1 and 2, which results in a frequency distribution of $\tau$ and $r_{\text {eff }}$ for each measurement point. As a result, $\tau$ and $r_{\text {eff }}$ are obtained (Fig. 10).

The 2-D frequency distributions are used to calculate the normalized mean values $(\bar{p})$ and standard deviations $\left(\sigma_{p}\right)$ of $\tau$ and $r_{\text {eff }}$ for each time stamp of measurement along the flight track (see Eqs. 3 and 4):

$\bar{p}=\sum_{i=1}^{N} w_{i} \cdot p_{i}$

$\sigma_{p}=\sqrt{\sum_{i=1}^{N} w_{i} \cdot\left(p_{i}-\bar{p}\right)^{2}} ;$

here, $p$ stands for the properties $\tau$ and $r_{\text {eff }}, w_{i}$ is the normalized frequency distribution albedo (see Fig. 6c), $N$ indicates the number of combinations of albedo values, $\bar{\tau}$ and $\bar{r}_{\mathrm{eff}}$ are the mean values, and $\sigma_{\tau}$ and $\sigma_{r_{\text {eff }}}$ represent the standard deviation of the statistical retrieval. These standard deviations 

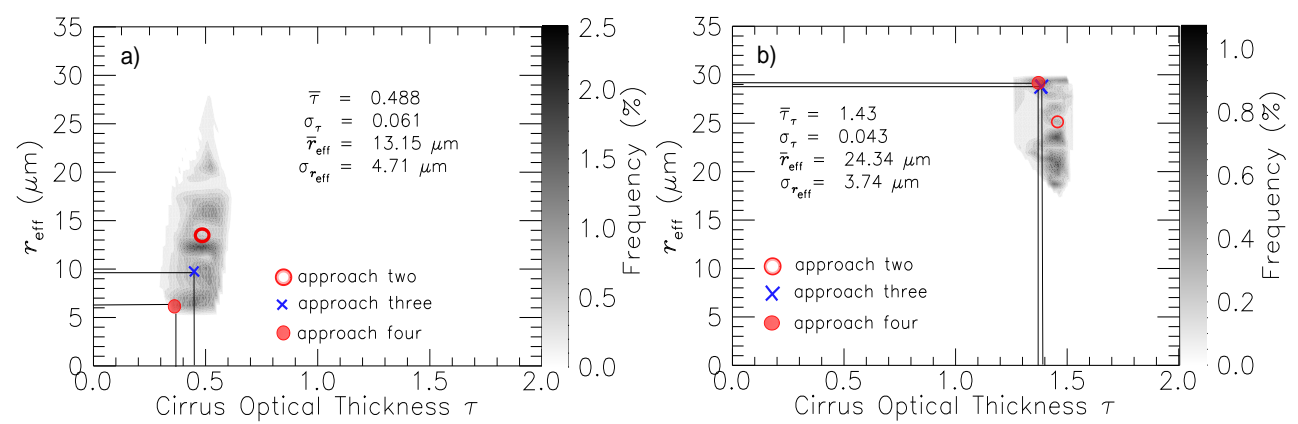

Fig. 11. Frequency distribution of $\tau$ and $r_{\text {eff }}$ of two HALO-SR measurement examples (a) $40601 \mathrm{~s}$, (b) $40795 \mathrm{~s}$ for the flight of 4 November 2010 using approach 2 . The red filled dot indicates the result for assuming the mean surface albedo of approach 4 compared to retrieval using the frequency distribution of approach 2 values (blank dot). The blue cross indicates the retrieval assuming the mean albedo given within a $10 \mathrm{~km}$ footprint below the plane for the measurement time stamp (approach 3)

quantify the effects of surface albedo heterogeneity on the retrieval of $\tau$ and $r_{\text {eff. }}$

\section{Retrieval results}

Figure 11 displays the retrieved frequency distribution of $\tau$ and $r_{\text {eff }}$ next to the retrieval results using approach 3 and 4 for two measurement points along the flight track from 4 November 2010.

While the range of most likely $\tau$ is situated in a small band of solutions in both cases (between 0.3 and 0.6 for case a and 1.2 to 1.6 for case b) using surface albedo approach $1, r_{\text {eff }}$ retrieval results reveal a larger uncertainty in general. Additionally, the value range within the standard deviation differs for both examples (5-25 $\mu \mathrm{m}$ for case a and $17-30 \mu \mathrm{m}$ for case b). The 2-D surface albedo frequency distribution leads to uncertainties quantified by standard deviations below 0.1 for $\tau$. For $r_{\text {eff }}$ both examples show differences in the uncertainty less than $5 \mu \mathrm{m} . \tau$ and $r_{\mathrm{eff}}$ retrieved by the standard retrieval assuming the mean surface albedo (approach 4) of the distribution under- or overestimate both $\tau$ and $r_{\text {eff }}$ compared to the statistical retrieval due to a nonlinear impact of the surface albedo on radiative transfer (Fig. 6a and b). A similar result can be observed when retrieving $\tau$ and $r_{\text {eff }}$ assuming the mean surface surface albedo within a $10 \mathrm{~km}$ footprint allocated below the plane for these two time stamps (approach $3)$.

A time series of $\sigma_{\tau}$ and $\sigma_{r_{\text {eff }}}$ along the flight path applying approach 1 is plotted as vertical red bars in Fig. 12a $\left(\sigma_{\tau}\right)$ and $\mathrm{b}\left(\sigma_{r_{\text {reff }}}\right)$. Accordingly, $\sigma_{\tau}$ and $\sigma_{r_{\text {eff }}}$ of approach 2 are plotted as black bars. In addition, retrieval results of approaches 3 and 4 are displayed. Blue squares indicate the retrieval results for $\tau$ and $r_{\text {eff }}$ applying approach 3, and the retrieval results applying approach 4 are illustrated as green diamonds. Concurrent HSRL measurements of $\tau$ are plotted as open triangles. The investigated cirrus shows mean optical thickness values between 0.3 and 1.5 with deviations for the retrieved $\tau$ lower than 0.1 (Fig. 12a). The deviation between
HSRL optical thickness and retrieved values from HALOSR measurements ranges between 0.4 but is in $80 \%$ of all cases above the measurement uncertainty of $5 \%$ (for each instrument). $r_{\text {eff }}$ offers a large variation between the different retrieval approaches, leading to values between 5 and $30 \mu \mathrm{m}$, covering the whole range of $r_{\text {eff }}$ used in the created lookup tables. Values of 5 and $30 \mu \mathrm{m}$ for the approaches 3 and 4 are not displayed, as they indicate a value outside the boundaries. $\sigma_{r_{\text {eff }}}$ of the two statistical approaches ranges between \pm 3 and $\pm 7.5 \mu \mathrm{m}$, as shown in Fig. 12b. For the investigated cirrus (4 November 2010), the horizontal structure or temporal variability is next to that reproduced by both instruments despite the mentioned bias.

Figure 13a shows the visible-wavelength (VIS) (B1) surface albedos for the four retrieval approaches of the corresponding time frame illustrating the variations of surface albedo especially the retrieval of $\tau$ is affected of. The VIS (B1) surface albedo ranges between 0.03 and 0.08 . Beside the constant value (green) created by approach 4 , the allocation of surface albedo below the plane using approach 3 offers the largest variation (blue) compared to the approaches using frequency distributions (red and black) with a variation of 0.04. Considering all approaches based on HALO-SR measurements, the surface albedo bias between the approaches leads to a maximum variation of $\tau$ of 0.2 including $\sigma_{r_{\text {eff }}}$. The corresponding NIR (B6) surface albedo mostly affecting the retrieval of $r_{\text {eff }}$ is displayed in Fig. 13b. A higher variation compared to the VIS (B1) surface albedo can be observed, as the surface albedo ranges between 0.09 and 0.21 , with a maximum deviation of 0.08 between the approaches. This leads to a maximum variation of $20 \mu \mathrm{m}$ within the retrieved $r_{\text {eff }}$.

While the 4 November 2010 time series features a continuous cirrus layer, Fig. 14 illustrates a more heterogeneous cirrus on 3 November 2010. As $\tau$ retrieved from the HSRL is based on the extinction between 7 and $13 \mathrm{~km}$ only, the approaches based on the HALO-SR measurements are based on the measurement signal including reflected radiation of a cloud in $5 \mathrm{~km}$ altitude. Thus, as shown in Fig. 14a, the 

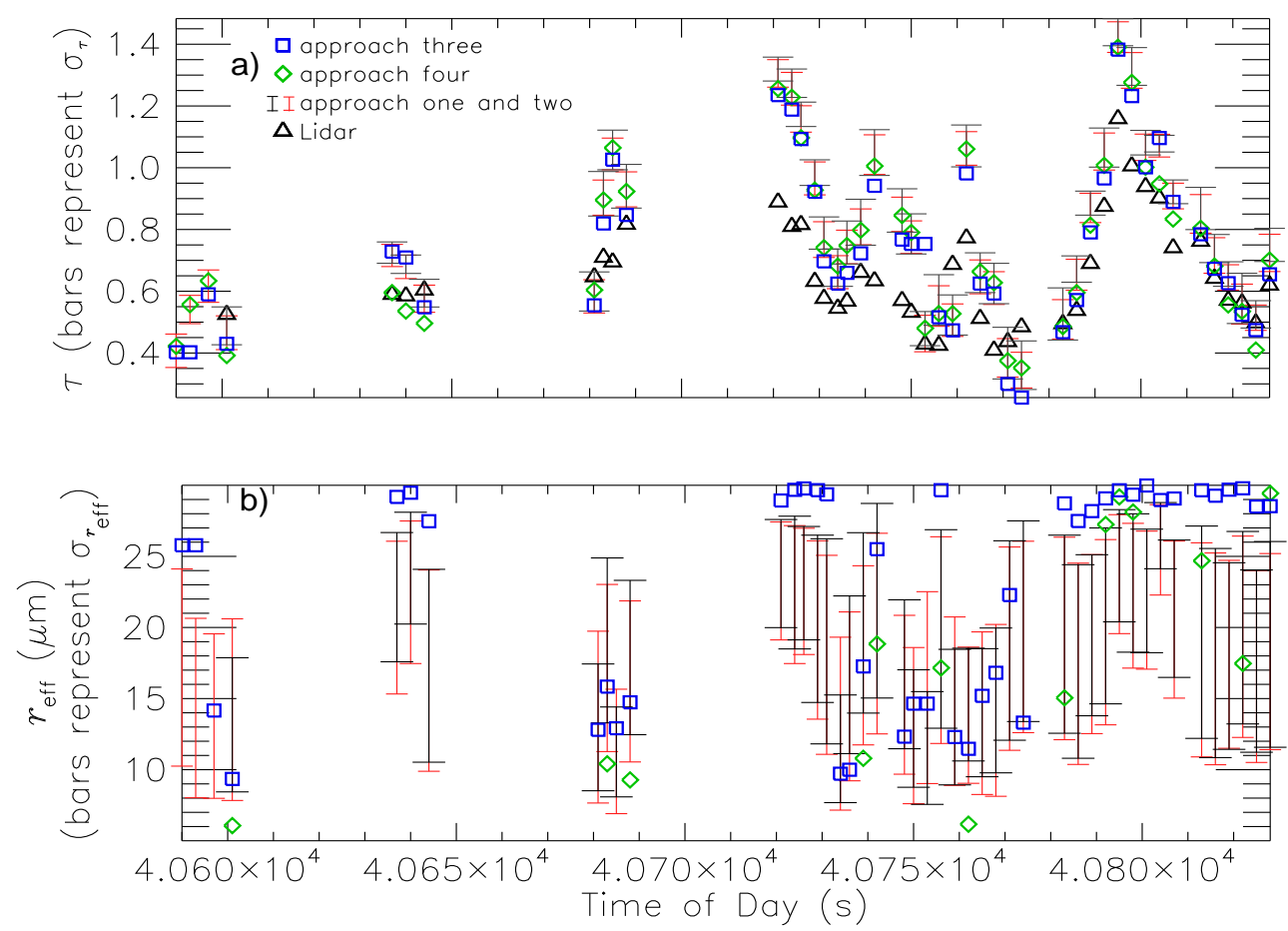

Fig. 12. (a) Comparison between $\sigma_{\tau}$ of retrieval approach using approach 1 (red bars), approach 2 (black bars), $\tau$ of standard retrieval using approach 3 (blue squares), $\tau$ of retrieval using approach 4 (green diamonds) derived from HALO-SR measurements, and $\tau$ derived from the HSRL (triangles) for the 4 November 2010 flight. Gaps are caused by roll movements of the plane, where nadir measurements could not be obtained. (b) $\sigma_{r_{\text {reff }}}$ and $r_{\text {eff }}$ of the corresponding time. Time is given in UTC.
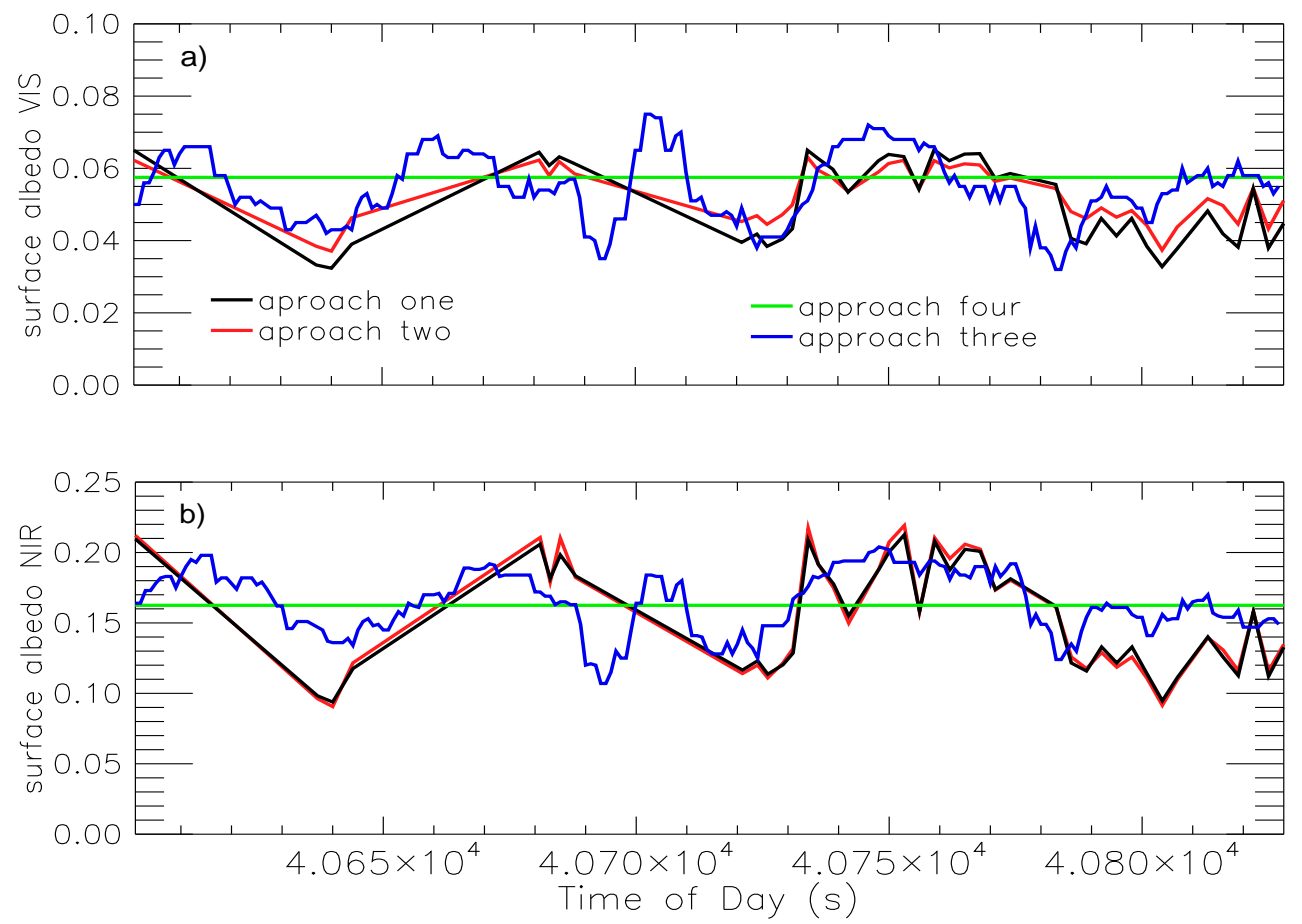

Fig. 13. (a) VIS surface albedo of time series corresponding to retrieval of 4 November. (b) NIR surface albedo of time series corresponding to retrieval of 4 November. Time is given in UTC. 

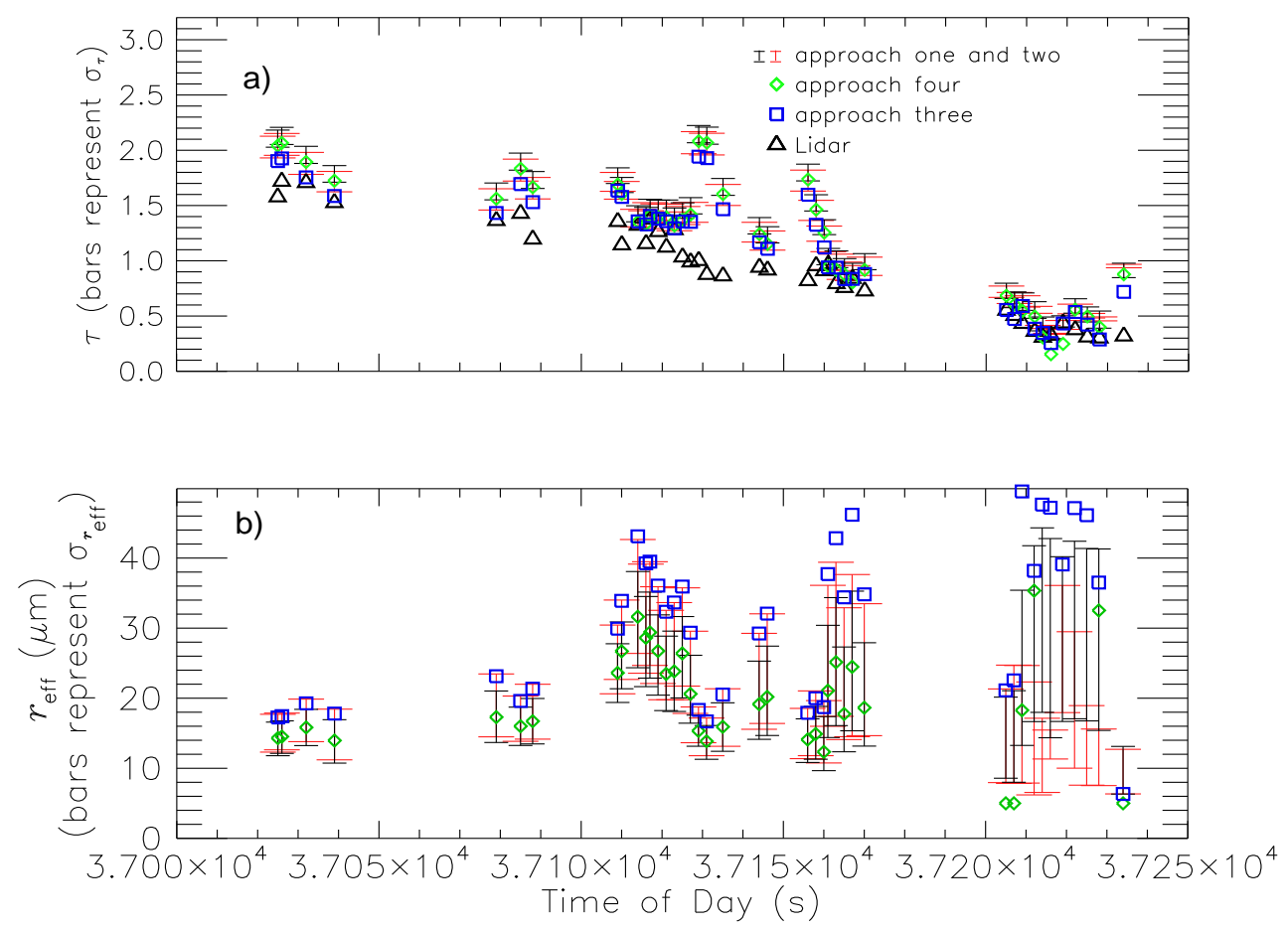

Fig. 14. Same as Fig. 12 but for 3 November 2010.

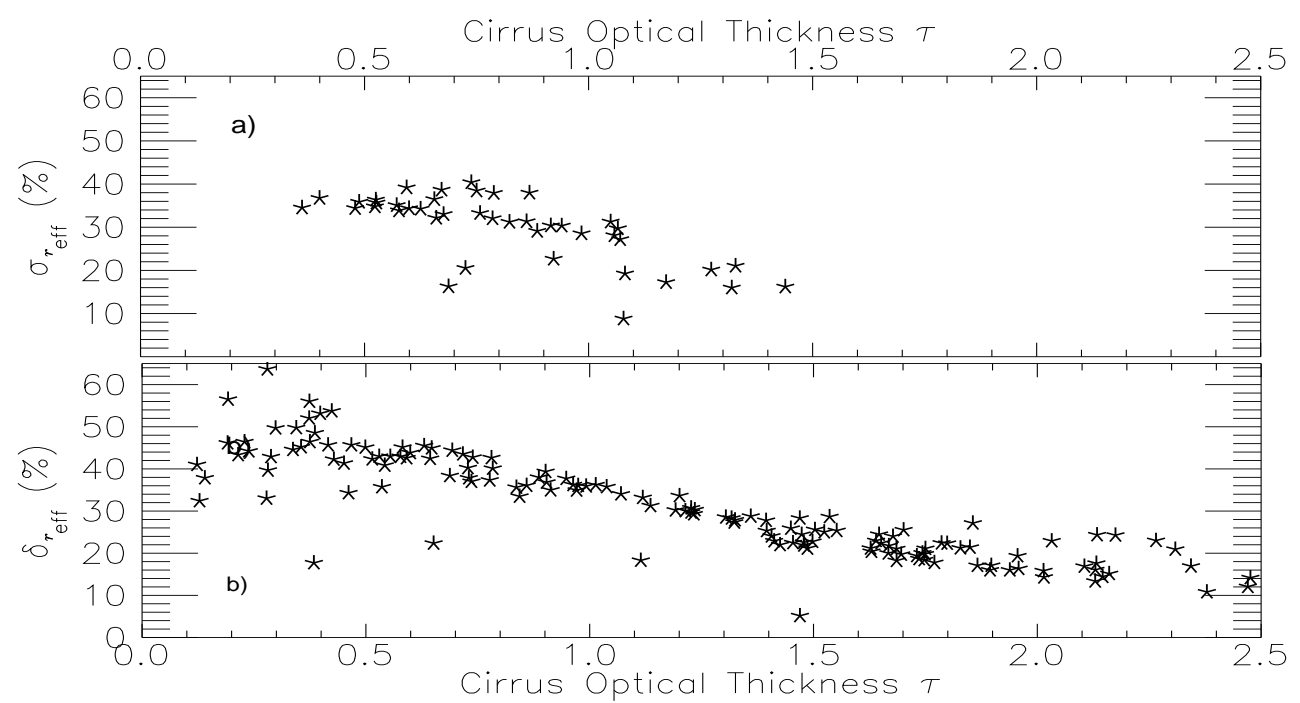

Fig. 15. Correlation between standard deviation from $r_{\text {reff }}$ and $\tau$ for the 4 November (a) and 3 November (b) time series derived of HALO-SR measurements using surface albedo approach 2.

HALO-SR-based retrieval approaches lead to higher values for $\tau$ compared to values derived by the HSRL. Nevertheless, the temporal course or horizontal variability is comparable. A continuous decrease of the investigated cloud leads to values of $\tau$ between 0.2 and 2.8 with standard deviations below 0.1 . $\bar{r}_{\text {eff }}$ varies between 9 and $40 \mu \mathrm{m}$, with a higher variation of standard deviation compared to the flight of 4 November. Comparing the different approaches, the deviation of $\tau$ is be- low 0.4. Regarding $r_{\text {eff }}$, an increasing variation of values can be observed.

The comparison of the results of both days indicates that the variation of retrieved $r_{\text {eff }}$ is proportional to the optical thickness; this comparison is shown in a correlation plot in Fig. 15. The $100 \mathrm{~km}^{2}$ surface albedo distribution (approach 2) is used as reference here. For both time series, $\sigma_{r_{\text {eff }}}$ decreases with increasing $\tau$, 


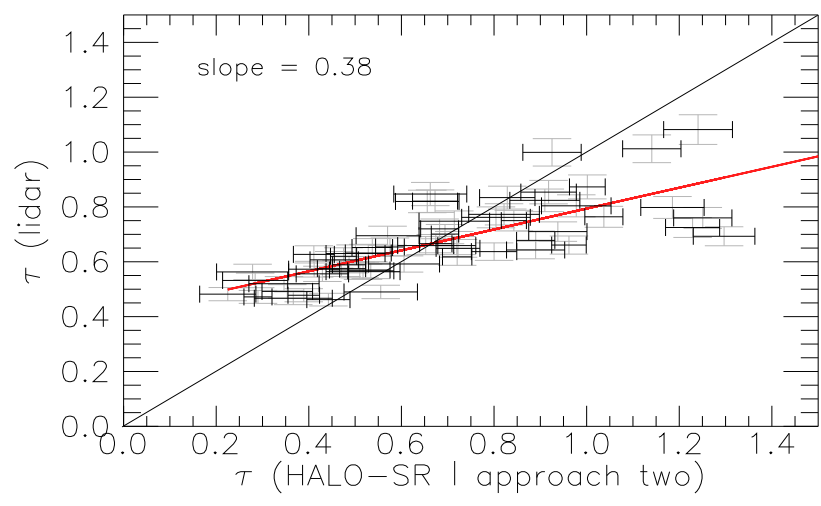

Fig. 16. Comparison of $\tau$ retrieved by the HSRL- and HALO-SRbased surface albedo approach 2 for 4 November 2010. For the calculations, a parametrization simulating a mixture of ice crystal shapes is used.

indicating a systematic relationship between both quantities. For 4 November (Fig. 15a) the standard deviation reaches values between 40 and $50 \%$ for $\tau=0.5$, while it decreases to standard deviations of $20 \%$ for $\tau=1.5$. A similar correlation is observed for 4 November (Fig. 15b), with the coefficient of variation decreasing to $10 \%$ for $\tau=2.5$ in Fig. $15 \mathrm{~b}$ as higher values for $\tau$ occurred here. Furthermore, the bias to the retrieval approaches 3 and 4 not using frequency distributions is reduced. The reason for the decreasing standard deviation and reduced bias is the decreasing influence of the surface albedo with increasing $\tau$ when less radiation is transmitted through the cirrus, as shown in Fig. 8. Unlike the small deviations for the retrieved $\tau$, as expected the results for $r_{\text {eff }}$ offer a high sensitivity to the values for $\tau$.

\section{Systematic and microphysical uncertainties}

\subsection{Shape effects}

As shown in Key et al. (2002), ice crystal shape has an influence on the retrieved cirrus optical properties, with maximum shape-induced differences of up to $60 \%$ for $\tau$ and about $20 \%$ for $r_{\text {eff }}$. Here, especially the optical thickness retrieval is affected due to the reflected radiation in the visible wavelength range. While multiple scattering or a highly variable crystal distribution smearing out possible shape effects play a minor role due to the thin cirrus investigated here, the dominating single-scattering effects justify a study of the shape effects on the retrieval.

To investigate systematic differences between HSRL and HALO-SR measurements, the optical thickness derived from HSRL is compared to the retrieval based on approach 2 (utilizing the $100 \mathrm{~km}^{2}$ surface albedo distribution) for a mixture of different ice crystals in a scatter plot in Fig. 16.

Here the results of both methods do not agree within the uncertainty bars for most of the measurements. For low opti- cal thicknesses $(\tau \leq 0.5)$, the results derived by the HALOSR retrieval are lower than the HSRL data. With increasing $\tau$, the opposite is observed indicated by a slope of 0.38 . One possible reason is the influence of the ice crystal shape. Nonspherical ice crystals in cirrus considered in all radiative transfer simulations shown so far have been represented in the simulations by an ice crystal parametrization from Baum et al. (2007), where the bulk optical properties represent a mixture of different crystal shapes. To investigate the influence of these shape assumptions, all simulations are repeated by applying different ice crystal shape parametrizations. Four different shapes are taken into account: plates, droxtals, solid columns and aggregates (Yang et al., 2000). The results are presented in Fig. 17. In general there is still an overestimation of $\tau$ for all shapes. Considering the slopes, the crystal shape affects the result for $\tau \geq 0.5$ with increasing magnitude. Although not shown, this is only valid as long as multiplescattering effects continue to play a minor role. The result for droxtal shape shows the largest difference compared to the HSRL (slope $=0.38$ ). Solid (slope $=0.51$ ) and hollow columns (slope $=0.56$ ) lead to a smaller deviation in comparison to the assumption of droxtals. However, the aggregate parametrization gives the best match within the available value range of $\tau$. This indicates that the most likely aggregate, ice crystals, were present in the observed cirrus during the time series of measurement, although no perfect agreement is obtained with a slope of 0.71 .

\subsection{Grid density}

Independent of the chosen crystal shape, a general over- or underestimation of $\tau$ is observed, which is caused by a systematic feature of the retrieval. Although the retrievals of $\tau$ and $r_{\text {eff }}$ are connected due to scattering at the VIS retrieval wavelength and absorption at the NIR retrieval wavelength, they are not necessarily orthogonally related for moderate and thin clouds as the NIR wavelength is also affected by scattering. In Fig. 18 three radiance grids following the approach of Nakajima and King (1990) for three different assumptions of the surface albedo at $650 \mathrm{~nm}(0.05,0.1,0.2)$ are displayed. Measurements are shown by triangles for a thin cloud and squares for a thick cloud. Changing the radiance grid from albedo $=0.15$ to albedo $=0.25$, the measurements for the thin cloud mostly fall outside the grid, which means no value is retrieved. For the thick cloud this does not occur as the grid covers a larger value range in this region. We counted the number of grids (albedo values) for which a retrieval could be applied. The total number is plotted against the optical thickness in Fig. 18b. For decreasing $\tau$, the number of solution grid points based on a surface albedo bin size of 0.005 , a $\tau$ value range of 0 to 25 with a bin size 1

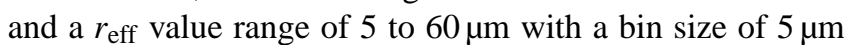
decreases as these solutions grids are nonlinear. The consequence is a slight over- or underestimation depending on the surface albedo distribution depending on the surface albedo 

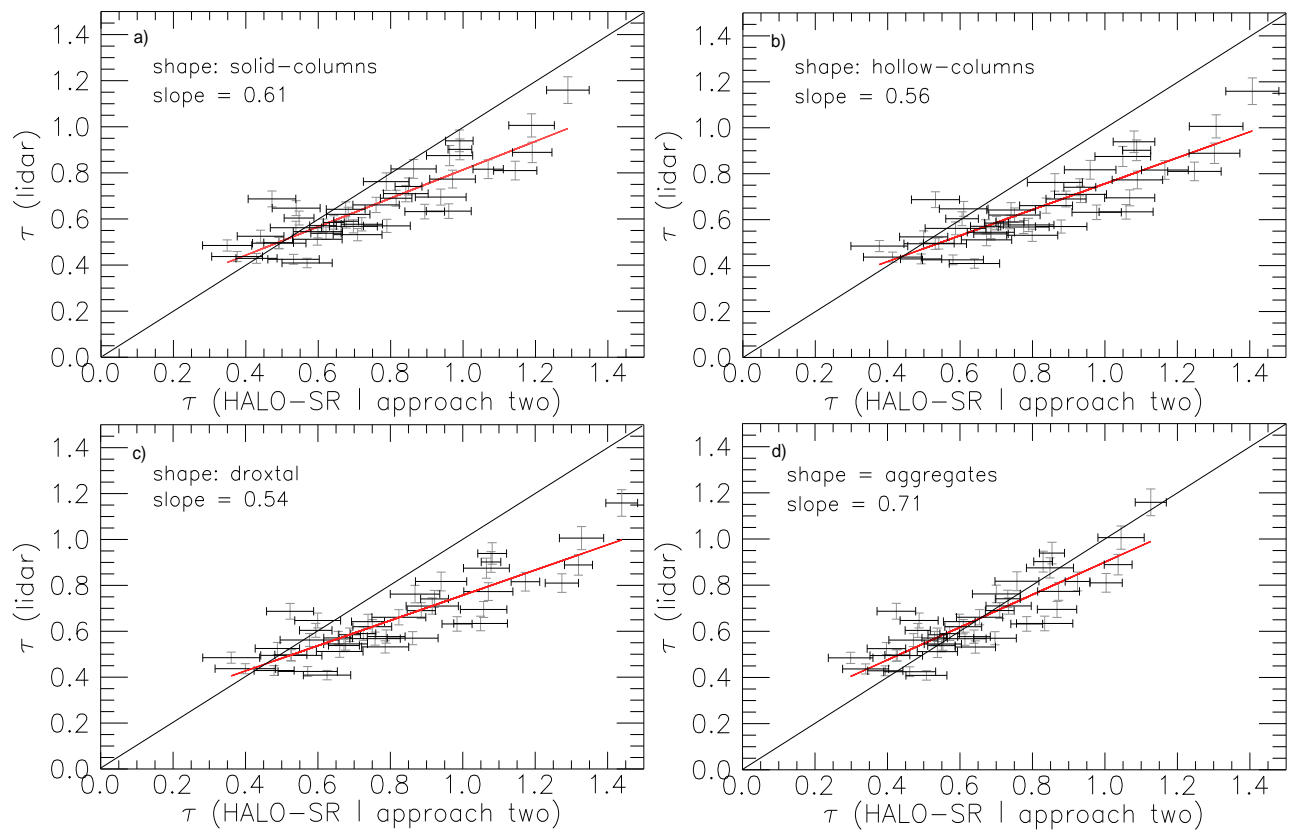

Fig. 17. Comparison of $\tau$ retrieved by the HSRL- and HALO-SR-based surface albedo approach 2 for different ice crystal shapes. Droxtals (c) show the biggest deviation, and hollow (b) and solid (a) columns slightly improve the slope. The best correlation is achieved for aggregates $(\mathbf{d})$. The influence increases with increasing $\tau$. For values below 0.5 , a systematic feature leading to an underestimation is beyond the shape influence.
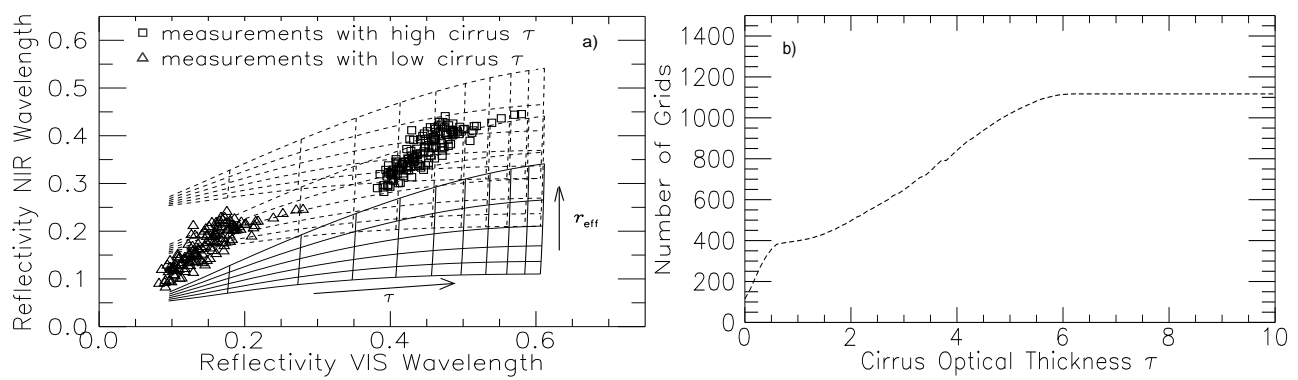

Fig. 18. (a) Illustration of systematic retrieval feature leading to an increasing underestimation of results for $\tau$ below 0.5 . Displayed are three radiance grids following the approach of Nakajima and King (1990) for three different assumptions of the surface albedo at $650 \mathrm{~nm}(0.05$, $0.15,0.25)$. Measurements for optically thick clouds are shown by squares. Triangles indicate measurements with low optical thickness. (b) Number of solution grids (lookup tables) based on homogeneous surface albedo distribution leading to a correct solution of the retrieval regulated by $\tau$.

distribution, affecting the retrieval of both $\tau$ and $r_{\text {eff. }}$ Figure 9 illustrates the systematic feature in the retrieval of $r_{\text {eff }}$ for different values of assumed surface albedo. Here an underestimation for assumed surface albedo values lower than the benchmark can be observed (black lines), which is caused by the systematic retrieval feature only, as different surface albedo assumptions lead to similar results.

\subsection{Bidirectional reflectance distribution function (BRDF)}

Investigations up to this point have considered an isotropic reflecting surface that reflects and scatters equally into all directions. To analyze possible angular-dependent scattering effects, the spectral MODIS BRDF product from 1 November 2010 has been implemented into the retrieval featuring similar atmospheric conditions as well as comparable solar zenith angles to the surface albedo products used so far. This product covers an area of approximately $3600 \mathrm{~km}^{2}$, comparable to approach 1 using the MODIS albedo product introduced in Sect. 2.2. The BRDF algorithm makes use of 16-day multi-data to derive parameters describing the bidirectional reflectance function that relies on the weighted sum of three parameters to determine the reflectance (Schaaf et al., 2002). The algorithm consists of one isotropic parameter $\left(f_{\text {iso }}(\lambda)\right)$ 

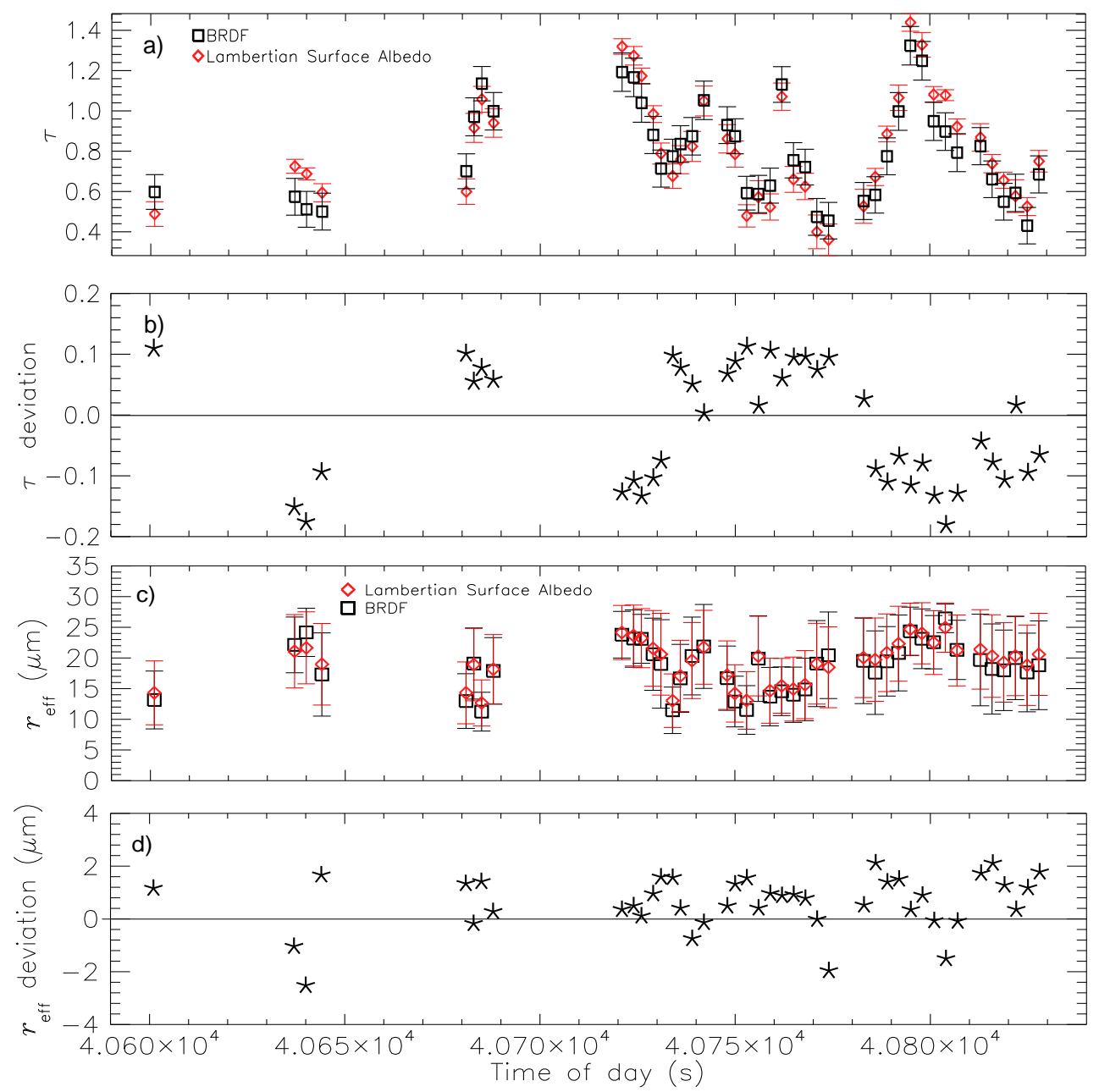

Fig. 19. (a) Comparison of mean values and standard deviations between retrieval using surface albedo approach 2 (HALO-SR) of $\tau$ using isotropic reflecting surface albedo (red diamonds) and BRDF (black squares) from the 4 November 2010 flight between 11:00 and 11:30 UTC.

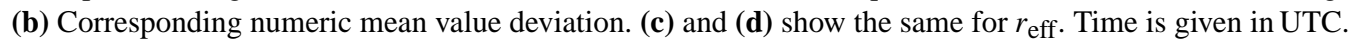

and two functions $\left(f_{\mathrm{vol}}(\lambda), f_{\text {geo }}(\lambda)\right)$ of viewing and illumination geometry, all of which are wavelength dependent. While the statistical retrieval using isotropic surface albedo relies on one dimension for the albedo value for each wavelength, the BRDF adds two more dimensions for each wavelength that has to be considered. The dimension of isotropic surface albedo was replaced by these three BRDF parameters in the lookup tables. This leads to an exponential increase in calculation times not feasible for all the statistical retrieval. As a consequence, the amount of MODIS pixels was reduced by choosing one-tenth random values out of the whole value range of surface BRDF. Tables 1 and 2 show mean values and standard deviations of the original and the reduced random-value BRDF weighting parameter $\left(f_{\text {iso }}, f_{\text {vol }}, f_{\text {geo }}\right)$ distribution for the $\mathrm{B} 1$ and $\mathrm{B} 6$ wavelength range, proving comparable statistics regarding the value ranges presented in Sect. 3.2.
Table 1. Statistical comparison between original and reduced random number BRDF distribution weighting parameters $\left(f_{\text {iso }}, f_{\mathrm{vol}}\right.$, $f_{\text {geo }}$ ) for the B1 wavelength range.

\begin{tabular}{ccccc}
\hline Parameter & \multicolumn{2}{c}{ Mean Value } & \multicolumn{2}{c}{ Standard Deviation } \\
\hline & BRDF & BRDF $_{\text {reduced }}$ & BRDF & BRDF $_{\text {reduced }}$ \\
\hline$f_{\text {iso }}(620-670 \mathrm{~nm})$ & 0.0659 & 0.0623 & 0.0205 & 0.0180 \\
$f_{\text {vol }}(620-670 \mathrm{~nm})$ & 0.0306 & 0.0274 & 0.0134 & 0.0113 \\
$f_{\text {geo }}(620-670 \mathrm{~nm})$ & 0.0105 & 0.0099 & 0.0046 & 0.0037 \\
\hline
\end{tabular}

The comparison between the retrieval of $\tau$ using Lambertian reflecting surface albedo and the BRDF is presented in Fig. 19a and b based on a mixture of different ice crystal shapes. Mean values of both $\tau$ retrievals generally agree as the deviations are within \pm 0.2 . The standard deviations do not exceed 0.1 for both retrievals. However, the standard deviations are slightly larger in some cases for the BRDF case, 
Table 2. Statistical comparison between original and reduced random number BRDF distribution weighting parameters $\left(f_{\text {iso }}, f_{\text {vol }}\right.$, $\left.f_{\text {geo }}\right)$ for the B6 wavelength range.

\begin{tabular}{ccccc}
\hline Parameter & \multicolumn{2}{c}{ Mean Value } & \multicolumn{2}{c}{ Standard Deviation } \\
\hline & BRDF & BRDF $_{\text {reduced }}$ & BRDF & BRDF $_{\text {reduced }}$ \\
$f_{\text {iso }}(1628-1652 \mathrm{~nm})$ & 0.1857 & 0.1995 & 0.0408 & 0.0379 \\
$f_{\text {vol }}(1628-1652 \mathrm{~nm})$ & 0.0999 & 0.0981 & 0.0341 & 0.0331 \\
$f_{\text {geo }}(1628-1652 \mathrm{~nm})$ & 0.0207 & 0.0232 & 0.0094 & 0.0095 \\
\hline
\end{tabular}

which is caused by the larger amount of surface albedo values affecting the retrieval. Nevertheless, the influence of the BRDF on the retrieval of $\tau$ is minimal in comparison to the general influence of the surface albedo. The influence of the BRDF on the retrieval of $r_{\text {eff }}$ is presented in Fig. 19c and d. The retrieval of $r_{\text {eff }}$ leads to comparable results as the numeric deviations between both mean value retrievals range between $\pm 2.5 \mu \mathrm{m}$. The standard deviations of the BRDF retrieval show no significant differences to the Lambertian retrieval. The slightly larger standard deviations in some cases in the $\tau$ retrieval are not reproduced for the $r_{\text {eff }}$ retrieval of this time series. This states that the BRDF influence is minimal for the $r_{\text {eff }}$ as the directional scattering is most significant at a lower wavelength with regard to the cirrus optical thickness.

\section{Conclusions}

The influence of surface albedo variability on the retrieval of cirrus properties ( $\tau$ and $r_{\text {eff }}$ ) was investigated based on airborne HALO-SR measurements. Four approaches of surface albedo variability were used in a standard retrieval to quantify the variations of $\tau$ and $r_{\text {eff }}$ with regard to the assumption of surface albedo. For the cirrus optical thickness, the standard deviation mostly is below 0.1 for the approaches using frequency distributions of surface albedo. In comparison to the two approaches not using frequency distributions, the bias between the approaches reaches values smaller than 0.3 at most. On the one hand, this shows that the presented method using frequency distributions of surface albedo is suitable for retrieving cloud optical thicknesses over heterogeneous surface situations when the surface albedo is not known. On the other hand, the possible error created by utilizing the wrong surface albedo results in a variation of $\tau$ introducing a maximum of 0.3 . However, it has to be pointed out that these results apply only for the cirrus and surface albedos analyzed here. In general, the uncertainty increases with increasing width of the surface albedo distribution and thus increasing amount of surface types. In addition, the distribution itself is an important influencing factor, as an approach considering the distribution by weighting the result according to its occurrence frequency reduces the variability in comparison to an approach taking the surface types into account equally. As a consequence, the results depends on the surface albedo frequency distribution applied to the performed retrieval. Here, $\sigma_{\tau}$ and $\sigma_{r_{\text {eff }}}$ as parameters for characterizing the variation are one approach to illustrate retrieval variations according to the surface albedo distribution and may be changed by other statistical parameters according to the required probabilities. However, especially for high variabilities of surface albedo on a small scale, the potential for errors induced by wrong surface albedo assumptions cannot be neglected. Using surface albedo distributions in the retrieval may therefore be a feasible method for excluding possible errors, as it prevents the calculation of considerable numeric deviations.

For the effective radius retrieval the sensitivity to surface albedo depends on the cirrus optical thickness. The smaller the cirrus optical thickness, the larger the uncertainty of the effective radius with regard to the surface albedo heterogeneity. This also applies for the retrieval approaches excluding frequency distributions of surface albedo, because variations, both amongst each other and in regard to the approaches including frequency distributions of surface albedo, increase with decreasing cirrus optical thickness. Uncertainties caused by the ice crystals shape are observed for optical thicknesses larger than 0.5 (in regard to the optical thicknesses investigated here) and can be neglected for lower values. This limit is also applicable to systematic issues relating to the retrieval approach itself affecting the retrieval for optical thicknesses below 0.5. It is induced as the retrieval wavelengths in VIS and NIR spectral range are not necessarily connected orthogonally. A comparison with the optical thickness from HSRL measurements showed best agreement for aggregates. Compared to the HSRL, the factor between lidar and HALO-SR using the best and worst crystal shape assumption is about 2 . This ice crystal shape influence is supported by the corresponding literature, introducing uncertainties of up to $60 \%$ for $\tau$ and $20 \%$ for $r_{\text {eff }}$ (Key et al., 2002). The influence of the bidirectional reflectance distribution function (BRDF) is minimal as it shows no significant differences to the use of isotropic reflecting surface albedo. Liang et al. (2002) support these results on the basis of satellite retrievals of surface albedo. One possible source of uncertainty needs to be investigated in the future. At this point the radiative transfer calculations are performed in a 1-D approach leaving out possible 3-D atmospheric scattering effects. This needs to be investigated by means of 3-D radiative transfer modeling, which will be addressed in further investigations. 
Acknowledgements. We are grateful for the funding of projects WE 1900/21-1, WE 1900/22-1 and WE 1900/24-1 and BO 1580/4-1 provided by the Deutsche Forschungsgemeinschaft within the framework of HALO SPP 1294. Furthermore, we appreciate the assistance from DLR-FX during the campaign, and we highly appreciate the comprehensive input given by the reviewers.

Edited by: M. Krämer

\section{References}

Baum, B. A., Heymsfield, A. J., Yang, P., and Thomas, S. M.: Bulk scattering models for the remote sensing of ice clouds. Part I: Microphysical data and models., J. Appl. Meteor., 44, 1885-1895, 2005.

Baum, B. A., Yang, P., Nasiri, S., Heidinger, A. K., Heymsfield, A., and Li, J.: Bulk scattering properties for the remote sensing of ice clouds. Part III: High-resolution spectral models from 100 to $3250 \mathrm{~cm}^{-1}$, J. Appl. Meteor., 46, 423-434, 2007.

Bierwirth, E., Wendisch, M., Jakel, E., Ehrlich, A., Schmidt, K. S., Stark, H., Pilewskie, P., Esselborn, M., Gobbi, G. P., Ferrare, R., Muller, T., and Clarke, A.: A new method to retrieve the aerosol layer absorption coefficient from airborne flux density and actinic radiation measurements RID E-7433-2010, J. Geophys. Res.-Atmos., 115, D14211, doi:10.1029/2009JD013636, 2010.

Eichler, H., Ehrlich, A., Wendisch, M., Mioche, G., Gayet, J.F., Wirth, M., Emde, C., and Minikin, A.: Influence of ice crystal shape on retrieval of cirrus optical thickness and effective radius: A case study, J. Geophys. Res., 114, D19203, doi:10.1029/2009JD012215, 2009.

Esselborn, M., Wirth, M., Fix, A., Tesche, M., and Ehret, G.: Airborne high spectral resolution lidar for measuring aerosol extinction and backscatter coefficients, Appl. Opt., 47, 346-358, 2008.

Gao, B. C., Han, W., Tsay, S. C., and Larsen, N. F.: Cloud detection over the Arctic region using airborne imaging spectrometer data during the daytime, J. Appl. Meteorol., 37, 1421-1429, doi:10.1175/1520-0450(1998)037<1421:CDOTAR>2.0.CO;2, 1998.

Hong, G., Yang, P., Gao, B. C., Baum, B. A., Hu, Y. X., King, M. D., and Platnick, S.: High cloud properties from three years of MODIS Terra and Aqua collection-4 data over the Tropics, J. Appl. Meteor. Clim.,, 46, 1840-1856, 2007.

Key, J. R., Yang, P., Baum, B. A., and Nasiri, S. L.: Parameterization of shortwave ice cloud optical properties for various particle habits, J. Geophys. Res., 107, 4181, doi:10.1029/2001JD000742, 2002.

Liang, S., Fang, H., Chen, M., Shuey, C., C. J., W., Daughtry, C., Morisette, J., Schaaf, C., and Strahler, A.: Validating MODIS land surface reflectance and albedo products: methods and preliminary results, Remote Sens. Environ., 83, 149-162, 2002.

Mayer, B. and Kylling, A.: Technical note: The libRadtran software package for radiative transfer calculations - description and examples of use, Atmos. Chem. Phys., 5, 1855-1877, doi:10.5194/acp-5-1855-2005, 2005.

Nakajima, T. and King, M.: Determination of the optical thickness and effective particle radius of clouds from reflected solar radiation measurements. Part I: Theory, J. Atmos. Sci., 47, 18781893, 1990.
Piironen, P. and Eloranta, E. W.: Demonstration of a high-spectralresolution lidar based on an iodine absorption filter, Opt. Lett., 19, 234-236, doi:10.1364/OL.19.000234, 1994.

Ricchiazzi, P., Yang, S., Gautier, C., and Sowle, D.: SBDART: A research and Teaching software tool for plane-parallel radiative transfer in the Earth's atmosphere, B. Am. Meteorol. Soc., 79, 2101-2114, 1998.

Rolland, P., Liou, K., King, M., Tsay, S., and McFarquhar, G.: Remote sensing of optical and microphysical properties of cirrus clouds using Moderate-Resolution Imaging Spectrometer (MODIS) channels: Methodology and sensitivity to physical assumptions, J. Geohys. Res., 105, 11721-11738, 2000.

Sassen, K., Wang, Z., and Liu, D.: Global distribution of cirrus clouds from CloudSat/Cloud-Aerosol Lidar and Infrared Pathfinder Satellite Observations (CALIPSO) measurements, J. Geophys. Res., 113, D00A12, doi:10.1029/2008JD009972, 2008.

Schaaf, C. B., Gao, F., Strahler, A. H., Lucht, W., Li, X. W., Tsang, T., Strugnell, N. C., Zhang, X. Y., Jin, Y. F., Muller, J. P., Lewis, P., Barnsley, M., Hobson, P., Disney, M., Roberts, G., Dunderdale, M., Doll, C., d'Entremont, R. P., Hu, B. X., Liang, S. L., Privette, J. L., and Roy, D.: First operational BRDF, albedo nadir reflectance products from MODIS, Remote Sens. Environ., 83, 135-148, 2002.

Wendisch, M., Müller, D., Schell, D., and Heintzenberg, J.: An airborne spectral albedometer with active horizontal stabilization, J. Atmos. Ocean. Technol., 18, 1856-1866, 2001.

Wendisch, M., Pilewskie, P., Pommier, J., Howard, S., Yang, P., Heymsfield, A. J., Schmitt, C. G., Baumgardner, D., and Mayer, B.: Impact of cirrus crystal shape on solar spectral irradiance: A case study for subtropical cirrus, J. Geophys. Res., 110, D03202, doi:10.1029/2004JD005294, 2005.

Wirth, M., Fix, A., Mahnke, P., Schwarzer, H., Schrandt, F., and G., E.: The airborne multi-wavelength water vapor differential absorption LIDAR WALES: system design and performance, Appl. Phys. B: Laser Opt., 96, 201-213, 2009.

Yang, P., Liou, K. N., Wyser, K., and Mitchell, D.: Parameterization of the scattering and absorption properties of individual ice crystals, J. Geophys. Res., 105, 4699-4718, 2000.

Yang, P., Tsay, S. C., Wei, H., Guo, G., and Ji, Q.: Remote Sensing of Cirrus Optical and Microphysical Properties from GroundBased Infrared Radiometric Measurements - Part I: A New Retrieval Method Based on Microwindow Spectral Signature, IEEE Geosci. Remote Sens. Lett., 2, 128-131, 2005.

Zhang, Z., Yang, P., Kattawar, G., Riedi, J., -Labonnote, L. C., Baum, B. A., Platnick, S., and Huang, H.-L.: Influence of ice particle model on satellite ice cloud retrieval: lessons learned from MODIS and POLDER cloud product comparison, Atmos. Chem. Phys., 9, 7115-7129, doi:10.5194/acp-9-7115-2009, 2009. 